\title{
Numerical Simulation of Free Surface Fluid Flows Through Porous Media by Using the Explicit MPS Method
}

\author{
Xiaosong Sun ${ }^{1} \mathbb{D} \cdot$ Maodong Sun ${ }^{2} \cdot$ Kazuya Takabatake ${ }^{2} \cdot$ Christopher C. Pain $^{3}$. \\ Mikio Sakai ${ }^{1}$
}

Received: 11 May 2018 / Accepted: 17 October 2018 / Published online: 2 November 2018

(c) The Author(s) 2018

\begin{abstract}
An extension of the explicit moving particle semi-implicit (MPS) method is proposed for simulating free surface flow through a porous structure object. The model formulation is based on the local volume averaging equations, and the porous drag is given by a general closure function. It can compute the macroscopic behaviors of incompressible flows in porous media. Specifically, the present study addresses numerical difficulties of existing MPS models in two respects, namely the conservation of macroscopic fluid volume and the well-balanced hydrostatic equilibrium where the fluid and porous media interpenetrate. Numerical tests are performed to examine the validity and accuracy of the proposed model, from which good agreements are found between the simulation results and validation data. In comparison with existing models, it is also demonstrated that the numerical techniques developed in this study are important to obtain consistent predictions of flow behaviors. Therefore, the present MPS method is shown to be suitable for modeling free surface flows interacting with porous structures.
\end{abstract}

Keywords Explicit MPS method · Free surface flow · Porous media · Local volume averaging

$凶 \quad$ Xiaosong Sun

song@dem.t.u-tokyo.ac.jp

$凶$ Mikio Sakai

mikio_sakai@n.t.u-tokyo.ac.jp

1 Resilience Engineering Research Center, School of Engineering, The University of Tokyo, 7-3-1 Hongo, Bunkyo-ku, Tokyo 113-8656, Japan

2 Department of Nuclear Engineering and Management, School of Engineering, The University of Tokyo, 7-3-1 Hongo, Bunkyo-ku, Tokyo 113-8656, Japan

3 Applied Modeling and Computation Group, Department of Earth Science and Engineering, Imperial College London, Prince Consort Road, London SW7 2BP, UK 


\section{Introduction}

The interaction between free surface flows and porous structures is crucial to many problems in marine and coastal engineering (Liu et al. 1999), engine design (Rahimi-Gorji et al. 2017), and bio-systems (Rahimi-Gorji et al. 2015, 2016). With the growing power of modern computers, the numerical simulation is expected to be useful in understanding and analyzing the flow behaviors. From an engineering perspective, discussions hereinafter will focus on the macroscopic modeling applicable to large-scale problems involving flow-porous structure interactions. In this approach, the focus is the macroscopic behaviors of the fluid, e.g., pressure drop, average velocity, and surface elevation through porous media. The exact pore structure of the solid is not resolved. Instead, the porosity (void fraction) is employed to describe the configuration of different phases, and the resisting force (drag) is expressed by empirical closures depending on local porosity and volumetric flow rate. For simplicity, the porous material is assumed to be isotropic and stationary, and the effect of capillary force is neglected.

Eulerian models based on the volume-of-fluid (VOF) method (Hirt and Nichols 1981) have been developed in a number of past studies. In the VOF method, governing equations are discretized and solved on a grid fixed in space, and a special color function is used to label numerical cells occupied by the liquid phase. Liu et al. (1999) modified the VOF method by adding the porosity and drag to the continuity and momentum equations. They carried out both numerical and experimental studies of various flow cases involving wave-porous structure interactions. Other VOF-based models follow similar methodology in general, see e.g., Hieu and Tanimoto (2006), Karim et al. (2009), and Higuera et al. (2014).

An alternative approach for modeling free surface flows consists in Lagrangian particle methods gaining attentions recently, e.g., the smoothed-particle hydrodynamics (SPH) (Monaghan 1994) and the moving particle semi-implicit (MPS) method (Koshizuka and Oka 1996). In those methods, the continuum is discretized by computational particles that move with the material velocity. In comparison with conventional Eulerian methods, those Lagrangian particle methods are completely mesh-free, which thus saves the time-consuming task of mesh generation. Moreover, they are also advantageous in treating large deformations of the free surface including nonlinear dynamics of breakup and coalescence, which could be encountered in various industrial and environmental flows (Gómez-Gesteira et al. 2005; Shibata and Koshizuka 2007; Sun et al. 2012; Takabatake et al. 2016). Lagrangian particle methods have also been coupled with the discrete element method (Cundall and Strack 1979) to simulate solid-liquid two-phase flows involving free surfaces (Sakai et al. 2012; Yamada and Sakai 2013; Sun et al. 2013, 2014). The major difference between the SPH method and the MPS method lies in the spatial discretization schemes. The SPH method is based on the so-called kernel approximation, which can be taken as convolution with a Gaussian-like function. The spatial gradient of a variable thus involves the derivative of the kernel function. On the other hand, the MPS method directly discretizes the gradient, divergence, and Laplacian operators by weighted difference models. Interested readers are referred to the literature cited in this paragraph for more details.

Capability of the SPH method for simulating free surface flow-porous structure interactions has been explored by past studies. Shao (2010) developed a two-dimensional incompressible SPH (ISPH) method in which the fluid pressure is solved via a modified pressure Poisson equation (PPE). The method was applied to simulating wave damping over porous beds. Akbari and Namin (2013) and Akbari (2014) also adopted the ISPH approach to study porous dam break flows reported in Liu et al. (1999) and wave overtopping on porous 
breakwater. Another two-dimensional ISPH method was described by Pahar and Dhar (2016), who specifically showed that their model was able to recover the displacement of fluid volume due to saturated solid matrix. A three-dimensional ISPH method is available by Aly and Asai (2015). Besides the ISPH approach, Ren et al. (2014) developed their model based on the weakly compressible SPH (WCSPH) method. Unlike the ISPH that needs implicit inversion of the PPE system matrix, the WCSPH accelerates the pressure calculation by explicit calculation via the equation of state (EOS). A problem of Ren et al. (2014) is that different sets of governing equations are employed for fluids outside and inside the porous media separately, which complicated the algorithm by extra matching conditions at the porous boundary. With respect to this point, their later work (Ren et al. 2016) seemed to be simplified substantially by using unified equations of motion.

To the authors' best knowledge, the MPS method has not been applied to the simulation of flows through porous media as much extensively as the SPH method. Pahar and Dhar (2017) described a two-dimensional implicit MPS model as a variant of their SPH implementation (Pahar and Dhar 2016). It is, however, not clear whether their MPS model could conserve the gross fluid volume in porous region. Therefore, the numerical modeling of flow-porous media interaction has not been established thoroughly in the MPS framework.

In the present study, a modified MPS model is developed to perform three-dimensional numerical simulations of free surface fluid flows through porous media. This model is based on the explicit MPS method developed through our previous studies (Sakai et al. 2012; Yamada and Sakai 2013; Sun et al. 2014), which is more efficient than the original MPS method. The method of local volume averaging (Anderson and Jackson 1967) is adopted to derive unified governing equations for fluid motions in both free region and porous medium. Particularly, two numerical problems are addressed by this study to improve the quality of simulation results. The first problem is related to the conservation of macroscopic fluid volume in porous media, which means the gross volume represented by a particle must dilate in accordance with its current porosity as dictated by the condition of incompressibility. The second problem concerns the consistency of hydrostatic equilibrium between fluid domains with different porosities; unphysical behaviors will transpire if this condition is not guaranteed. It seems that previous studies are not aware of those two points which are originalities by the current work.

The new MPS model is verified by comparing its results with a mesh-based VOF code (Sun and Sakai 2015, 2016). Numerical tests show that the improved treatments of volume conservation and momentum balance are crucial for obtaining accurate results, which cannot be achieved by using existing MPS models. Finally, the proposed model is validated by the porous dam break flow problem (Liu et al. 1999), for which the simulation results are in good agreement with experimental data. Hence, it is shown that the proposed MPS model can predict macroscopic flow behaviors through porous media accurately. With those fundamental problems clarified in this study, it is expected that the MPS method will become a powerful tool for modeling such flows in practical applications.

\section{Physical Model}

Provided that the porosity $\varepsilon$ is known, the governing equations of incompressible fluid motion based on a local volume averaging description are written as follows (Yamada and Sakai 2013; Sun et al. 2014):

$$
\frac{D \varepsilon}{D t}+\varepsilon \nabla \cdot \boldsymbol{u}=0
$$




$$
\varepsilon \frac{D \boldsymbol{u}}{D t}=-\frac{\varepsilon}{\rho} \nabla p+\frac{\varepsilon \mu}{\rho} \nabla^{2} \boldsymbol{u}+\varepsilon \boldsymbol{g}+\frac{\boldsymbol{f}_{\mathrm{d}}}{\rho} .
$$

Herein, $D / D t$ is the material derivative, $\boldsymbol{u}$ is the actual fluid velocity, $\rho$ is the physical fluid density, $p$ is the pressure, $\mu$ is the dynamic viscosity, $g$ is the external gravity, and $f_{\mathrm{d}}$ is the drag force. Those two equations correspond to the conservative laws of mass and momentum, respectively. If porosity $\varepsilon$ is constant, Eq. (1) falls back to the conventional divergence-free velocity condition for incompressible fluids. Similarly, Eq. (2) becomes the Navier-Stokes equation with a source term of the porous drag. In addition, although neglected in this study, turbulence model could be incorporated as in the previous study (Nakayama and Kuwahara 1999).

The volumetric drag force $\boldsymbol{f}_{\mathrm{d}}$ may be written in the general Darcy-Forchheimer form:

$$
\boldsymbol{f}_{\mathrm{d}}=-[A \boldsymbol{U}+B|\boldsymbol{U}| \boldsymbol{U}] .
$$

Herein, scalars $A$ and $B$ are two model parameters, and the Darcy velocity $\boldsymbol{U}=\varepsilon \boldsymbol{u}$ gives the volumetric flux within porous region. In this equation, the first term on the right-hand side is linear with respect to the velocity, corresponding to the viscous low Reynolds number regime. The second term in quadratic form of velocity represents the inertial effect, which contributes to the drag force under mediate and high Reynolds numbers. In this study, we mainly consider porous structures that are formed by packing of granular materials with effective mean particle diameter $d_{\mathrm{p}}$. Hence, the Ergun's equation (Ergun 1952) is employed to determine the model parameters:

$$
A=150 \frac{(1-\varepsilon)^{2}}{\varepsilon^{2}} \frac{\mu}{d_{\mathrm{p}}^{2}}, \quad B=1.75 \frac{(1-\varepsilon)}{\varepsilon^{2}} \frac{\rho}{d_{\mathrm{p}}} .
$$

In free domains where the porosity $\varepsilon=1$, it is easy to confirm that both $A$ and $B$ reduce to zero and the drag vanishes. Therefore, free region and porous region can be treated in a unified way.

\section{Numerical Method}

In this section, the original MPS method is introduced first and then extensions for modeling porous structures as special subdomains are discussed. Numerical difficulties arising from the MPS porous model are demonstrated and explained, for which proper solutions are suggested.

\subsection{The Explicit MPS Method}

In the framework of the MPS method, fluids are discretized by Lagrangian particles that can move in space according to the material velocity. The MPS particles act as computational points where the flow variables are defined. The MPS method uses the weight function $w(r)$ for spatial discretization, which is usually a radial function that decreases monotonically with the inter-particle distance $r$ and gets truncated at some cutoff distance. In this study, the weight function developed in our previous studies (Yamada and Sakai 2013; Sun et al. 2014) is employed:

$$
w(r)=\left\{\begin{array}{ll}
\frac{r_{e}}{r}+\frac{r}{r_{e}}-2 & r<r_{e} \\
0 & r \geq r_{e}
\end{array} .\right.
$$




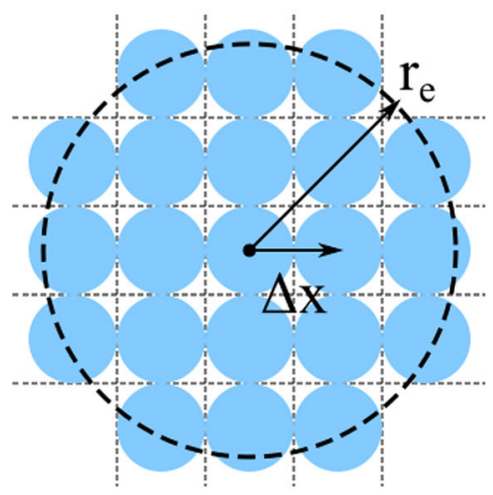

(a)

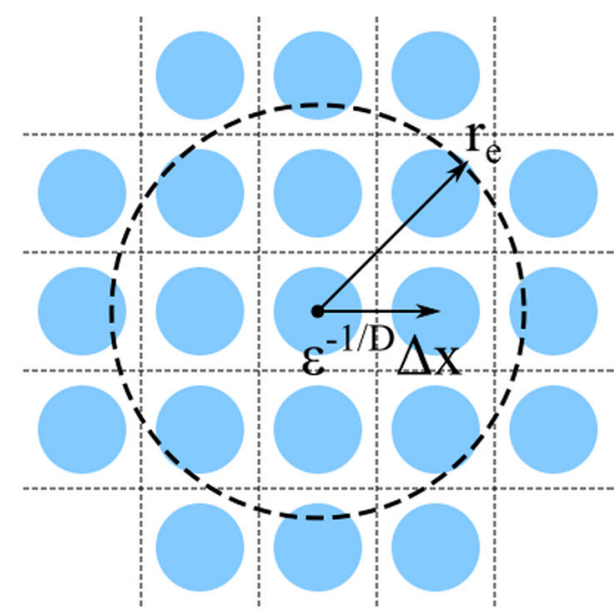

(b)

Fig. 1 Particle configurations of reference with different porosities: a original state $(\varepsilon=1)$ and $\mathbf{b}$ porous state $(\varepsilon<1)$. As the porosity decreases, the inter-particle distance increases reciprocally and the number of particles within the radius of influence $r_{e}$ reduces. The blue circles show original MPS particles with size equal to $\Delta x$, and the background grids show exact macroscopic volumes represented by them

Herein, $r_{e}$ is the influence radius beyond which the particle interaction decays to zero. The physical interactions between particles are thus limited within this compact distance. Numerically, its role is analogous to the grid spacing in mesh-based methods, which controls the spatial resolution. In the SPH and MPS methods, it is common to set $r_{e}$ with respect to the initial particle distance $\Delta x$ by a constant factor. Therefore, when particles are refined, the influence radius converges automatically. For the 3D simulations in this study, $r_{e}=3.1 \Delta x$ is used. This choice of influence radius is consistent with our previous study (Yamada and Sakai 2013). For solid-liquid flows, it is desired to use such a sufficiently large radius of MPS influence for numerical stability (Sun et al. 2014). It is also noted that, unlike the spline kernels widely employed in the SPH method, typical MPS weight function can be singular or non-differentiable at the origin (see e.g., Koshizuka and Oka 1996; Koshizuka et al. 1998; Shakibaeinia and Jin 2010; Yamada and Sakai 2013; Sun et al. 2014).

The mass conservation (or equivalently volume conservation for incompressible fluids) equation is treated in a Lagrangian manner by the MPS model. The local fluid density is related to the so-called particle number density. For the $i$ th particle, the particle number density $n_{i}$ is defined as the sum of weights of all the neighbor particles excluding the central particle itself:

$$
n_{i}=\sum_{j \neq i} w\left(r_{i j}\right)
$$

where the inter-particle distance is $r_{i j}=\left|\boldsymbol{x}_{j}-\boldsymbol{x}_{i}\right|$ with $\boldsymbol{x}_{i}$ and $\boldsymbol{x}_{j}$ denoting the particle coordinates. The characteristic value of particle number density (denoted by $n_{0}$ ) is obtained on a lattice of particles representing an ideal configuration. Such a representative configuration is shown in Fig. 1a, where the grid spacing is equal to the initial particle distance $\Delta x$. It is noted that the constant $n_{0}$ is computed for the virtual configuration during the preprocessing stage.

When the configuration of particles becomes disordered, the number density may deviate from $n_{0}$. It is thus desired to correct the defect to ensure the incompressibility of the fluid. In the 
original MPS method, this is achieved by implicitly solving the pressure Poisson equation, which is a complicated and computationally expensive procedure due to the unstructured nature of particle method. On the other hand, a weakly compressible model is adopted in the explicit MPS method. In this approach, the pressure is calculated via a modified equation of state (Monaghan 1994; Shakibaeinia and Jin 2010):

$$
p=\frac{\rho c^{2}}{\gamma}\left(\rho_{\text {rel }}^{\gamma}-1\right) \text {. }
$$

Herein, constant $\gamma=7$ is used for water. $\rho_{\text {rel }}$ is the relative density of the weakly compressible fluid equal to the ratio between the current number density and the characteristic value $n_{0}$. The artificial speed of sound $c$ is a numerical parameter used to limit the range of density fluctuation. In general, direct usage of the physical celerity is an overkill that will severely restrict the stable time step. The value of $c$ can be relaxed to accelerate the computation. According to Monaghan (1994) and Shakibaeinia and Jin (2010), it suffices to choose $c$ ten times as large as the characteristic flow velocity to guarantee density fluctuations are below $1 \%$, which is typically much smaller than the underlying physical sound speed. In this way, the explicit method can be evolved by affordable time steps. With proper choices of parameters, the explicit MPS method can significantly outperform the original model in efficiency (see e.g., Shakibaeinia and Jin 2010; Yamada et al. 2011 for benchmarks).

The differential operators in the momentum equation are approximated by discrete MPS operators based on weighted summations over neighbor particles. For example, the viscous term is discretized by using the Laplacian model (Koshizuka and Oka 1996):

$$
\left\langle\nabla^{2} \boldsymbol{u}\right\rangle_{i}=\frac{2 D}{n_{0} \lambda} \sum_{j \neq i}\left(\boldsymbol{u}_{j}-\boldsymbol{u}_{i}\right) w\left(r_{i j}\right) .
$$

Note that the angle brackets indicate a model approximation. Herein, $D$ is the dimension of space ( $D=3$ in this study) and $\lambda$ is the particle variance obtained from the same ideal configuration as $n_{0}$ :

$$
\lambda=\frac{1}{n_{0}} \sum_{j \neq i} r_{i j}^{2} w\left(r_{i j}\right) .
$$

In our previous studies (Yamada and Sakai 2013; Sun et al. 2014), the following form of pressure gradient model was proposed:

$$
\langle\nabla p\rangle_{i}=\frac{D}{n_{0, g}} \sum_{j \neq i}\left[\frac{p_{j}+p_{i}}{r_{i j}^{2}}\left(\mathbf{x}_{j}-\mathbf{x}_{i}\right) w_{g}\left(r_{i j}\right)\right] .
$$

It is noted that an alternative weight function $w_{g}$ is used here (see explanations in Sun et al. 2014):

$$
w_{g}(r)=\left\{\begin{array}{ll}
\frac{r_{e}}{r}-\frac{r}{r_{e}} & r<r_{e} \\
0 & r \geq r_{e}
\end{array} .\right.
$$

Consequently, the reference number density $n_{0, g}$ is derived for $w_{g}$ in the same way as described in the previous section. Apparently, the model is antisymmetric for the $(i, j)$ particle pair, which conserves momentum between interacting particles automatically (one can confirm this by swapping the particle labels and verifying their sum to be zero). Hence, Eq. (10) is referred to as the symmetric gradient model hereinafter.

In the following sections, the MPS method will be extended to take spatial porosity into account. 


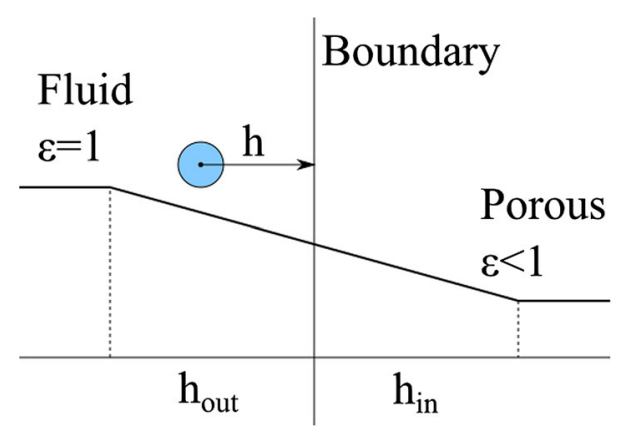

Fig. 2 Transition near the boundary between fluid and porous regions. In order to remove discontinuities, the porosity $\varepsilon$ is assumed to vary linearly within a short range $\left[h_{\mathrm{out}}, h_{\mathrm{in}}\right]$ across the porous interface. For a particle falling in this range, the porosity is determined by linear interpolation based on its distance $h$ from the boundary

\subsection{Porosity and Resistance}

Fixed porous structures are modeled as special numerical domains. For MPS particles inside the porous domain, their porosities are set to the prescribed value and the drag forces are evaluated by using Eqs. (3) and (4). The particles in free region have porosity of unity, and thus they are not affected by the drag, as commented previously. However, an MPS particle traveling across porous boundary undergoes a sudden jump in the porosity, which may give rise to numerical problems. Therefore, artificial margins are added on both sides of the porous boundary to guarantee a smooth transition. This is explained in Fig. 2, in which it is assumed that the porosity varies linearly within a short range $\left(h_{\text {out }}+h_{\text {in }}\right)$ from the boundary. For particle $i$ at distance $h$ from the porous boundary, the porosity is then determined by interpolation:

$$
\varepsilon_{i}=\varepsilon+\frac{h+h_{\text {in }}}{h_{\text {out }}+h_{\text {in }}}(1-\varepsilon) .
$$

In this paper, we have $h_{\text {out }}=h_{\text {in }}=2 \Delta x$ if not specified.

\subsection{Volume Conservation}

When liquids flow into the porous media, they can fill only the pore structures, while other parts are blocked by the solid matrix. From a macroscopic point of view, the displacement is equivalent to the expansion of the effective volume of the fluid, i.e.,

$$
\Delta V_{\varepsilon}=\frac{\Delta V}{\varepsilon},
$$

where $\Delta V$ is the original volume and $\Delta V_{\varepsilon}$ is the effective volume of the same amount of fluid inside the porous media. In the context of the MPS method, this implies a rarefied configuration of particles, as shown in Fig. 1. In Fig. 1a, the inter-particle distance is $\Delta x$ for the original state. On the other hand, the average distance in Fig. 1b where porosity $\varepsilon<1$ should be

$$
\Delta x_{\varepsilon}=\Delta x \cdot \varepsilon^{-1 / D} .
$$

Again, $D=3$ is the spatial dimension. As a result, there will be fewer particles within the influence radius, which consequently leads to deficiency in particle number density. Two possible solutions are known for this problem. The first approach is to employ variable 
influence radius scaling with the current inter-particle distance (Ren et al. 2016), of which the implementation is complicated. Thus, the second approach is adopted in this study by defining a proper mapping between the reduced particle number density $n_{\varepsilon}$ and the original value $n_{0}$ to enforce the conservation of fluid volume (Sun et al. 2014).

A simple assumption made by previous studies (Sakai et al. 2012; Yamada and Sakai 2013; Sun et al. 2013, 2014; Pahar and Dhar 2017) is that the particle number density is proportional to the current porosity. However, this is inexact even for regular configurations of MPS particles. We computed $n_{\varepsilon}$ for different porosities on aligned lattices (as shown in Fig. 1) and plotted their ratios against $n_{0}$ in Fig. 5 (blue circles). It is clearly seen that results are systematically lower than the analytical equation $\left(n_{\varepsilon} / n_{0}=\varepsilon\right)$ and their discrepancy enlarges as the porosity decreases. Provided that simple linear mapping fails for such regular states, it is no point to expect the performance on random particle configurations. This problem implies violation of the conservation of macroscopic fluid volume by existing MPS models.

In fact, the original form of the MPS particle number density may be understood as a discrete quadrature of the weight function excluding the possibly singular central part:

$$
n_{i}=\sum_{j \neq i} w\left(r_{i j}\right) \approx \int_{\Omega \backslash \Omega_{i}} w \mathrm{~d} \Omega,
$$

in which $\Omega$ denotes the volume normalized by the particle size. Figure 3 schematically illustrates the evaluation of particle number density. In this figure, neighbor particles (blue circles) covered within the influence radius are used in Eq. (15), while the central particle (yellow circle) is excluded from the computation due to the singularity of weight function. Given that the influence radius $r_{e}$ is fixed, the fraction of the area represented by the central particle increases as the porosity increases (equivalent to the expansion of inter-particle distance). From a physical perspective, excluding its contribution causes excessively rarefied particle distribution function. It would be thus favorable to take the weight of the central particle into account to improve the accuracy of density mapping. We thus derive an equivalent weight definition for the central particle to circumvent the singularity of the weight function [Eq. (5)].

Notice this singularity of Eq. (5) is weak in the sense that $w(r)$ is integrable in $2 \mathrm{D}$ and $3 \mathrm{D}$ spaces. This fact is also true for other MPS weights with $r^{-1}$ singularity (e.g., that proposed by Koshizuka and Oka 1996). For example, in two dimensions, the integral in radial direction is given by

$$
I(r)=\int\left[\frac{r}{r_{e}}+\frac{r_{e}}{r}-2\right] \cdot 2 \pi r \mathrm{~d} r=2 \pi r_{e}^{2}\left[\frac{q^{3}}{3}-q^{2}+q\right],
$$

where

$$
q \equiv r / r_{e} .
$$

Similarly, in three dimensions, the integral is obtained as

$$
I(r)=\int\left[\frac{r}{r_{e}}+\frac{r_{e}}{r}-2\right] \cdot 4 \pi r^{2} \mathrm{~d} r=4 \pi r_{e}^{3}\left[\frac{q^{4}}{4}-\frac{2 q^{3}}{3}+\frac{q^{2}}{2}\right] .
$$

We define the equivalent weight $\alpha$ for the core region within $r$ by taking the average of $I(r)$. In two dimensions, the result is

$$
\alpha(r)=\frac{I(r)}{\pi r^{2}}=\frac{2 q}{3}+\frac{2}{q}-2 .
$$




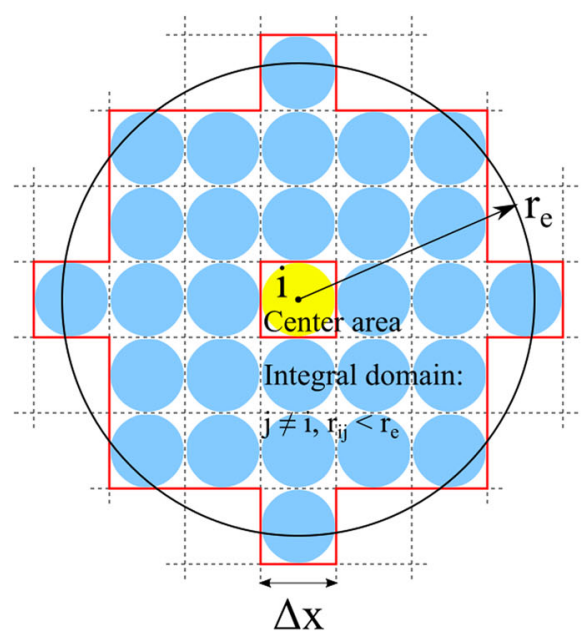

(a)

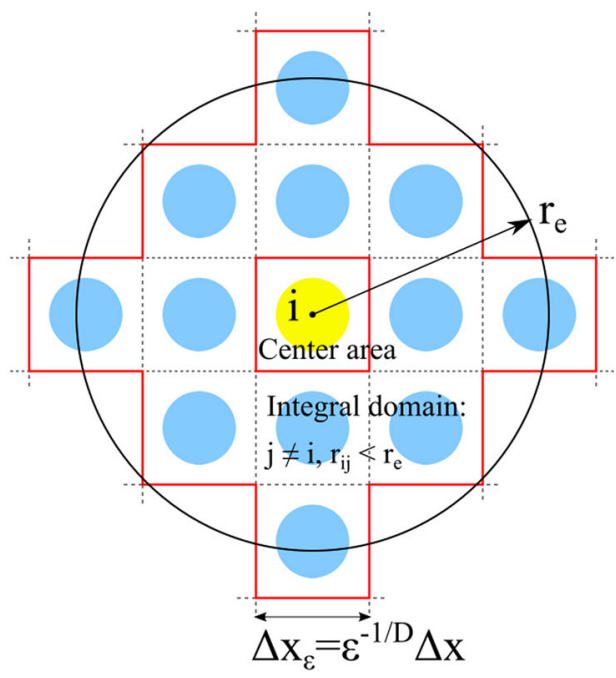

(b)

Fig. 3 Integration of particle number density around central particle in $\mathbf{a}$ original state with $\varepsilon=1$ and $\mathbf{b}$ porous state with $\varepsilon=0.44$. The yellow circle shows the central particle, and blue circles are its neighbors. The influence radius indicated by the large black circle is fixed to $r_{e}=3.1 \Delta x$. Neighbor particles accommodated within the range of influence are outlined by red segments, from which the center area is excluded. It is clearly seen that in porous state fewer neighbor particles are involved in the particle number density computation and the contribution of central particle (i.e., the center area part) becomes more significant

The three-dimensional central weight is

$$
\alpha(r)=\frac{I(r)}{(4 / 3) \pi r^{3}}=\frac{3 q}{4}+\frac{3}{2 q}-2 .
$$

The integral limit $r$ depends on the porosity, for which a natural choice is to use the expanded particle distance (Fig. 3b):

$$
\begin{aligned}
r & =\frac{1}{2} \Delta x_{\varepsilon}=\frac{1}{2} \frac{\Delta x}{\varepsilon^{1 / D}} \\
q & =\frac{r}{r_{e}}=\frac{1}{2 \varepsilon^{1 / D}} \frac{\Delta x}{r_{e}} .
\end{aligned}
$$

By inserting Eq. (21) into Eq. (19) or (20), the factor $\alpha$ can be computed straightforwardly from the porosity. Figure 4 plots the equivalent weight $\alpha$ against porosity $\varepsilon$ with influence radius $r_{e} / \Delta x=3.1$. The weight of the nearest neighbor particle is also plotted for comparison.

By taking the central weight into account, the ratio of particle number density is corrected as

$$
n_{\mathrm{rel}}=\frac{n_{\varepsilon}+\alpha_{\varepsilon}}{n_{0}+\alpha_{0}}
$$

where $\alpha_{\varepsilon}$ and $\alpha_{0}$ are the central weights calculated for the porous configuration and the original configuration, respectively. Figure 5 compares the corrected results with those without correction [i.e., neglecting both $\alpha$ terms in Eq. (22)]. It is confirmed that the particle den- 


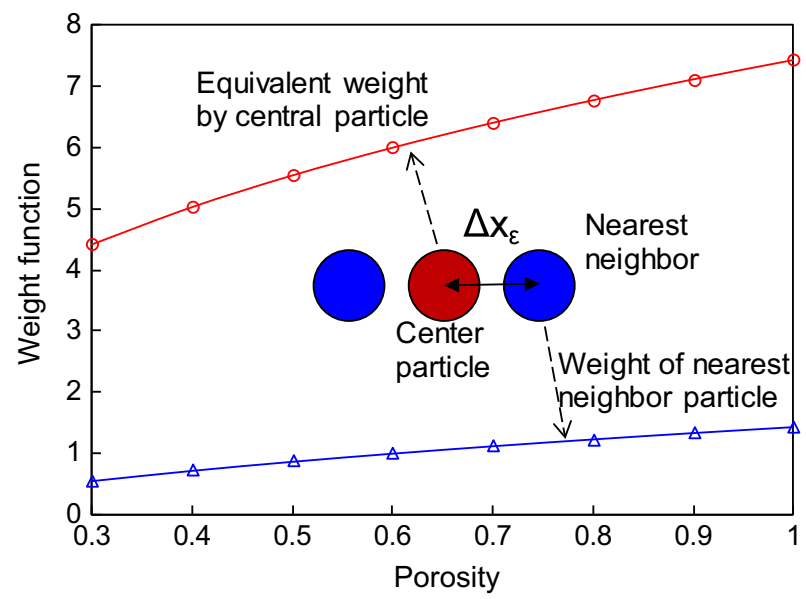

Fig. 4 Plot of the equivalent central weight with influence radius $r_{e}=3.1 \Delta x$. The weights of the nearest neighbor particle (i.e., the particle lying just next to the central particle on the grid) are also given as a guide of magnitude

Fig. 5 Relative particle number density for reference states with different porosities. Analytically, a linear relationship is expected. The relative number density computed deviates from the theory as porosity decreases if the central weight correction is not used

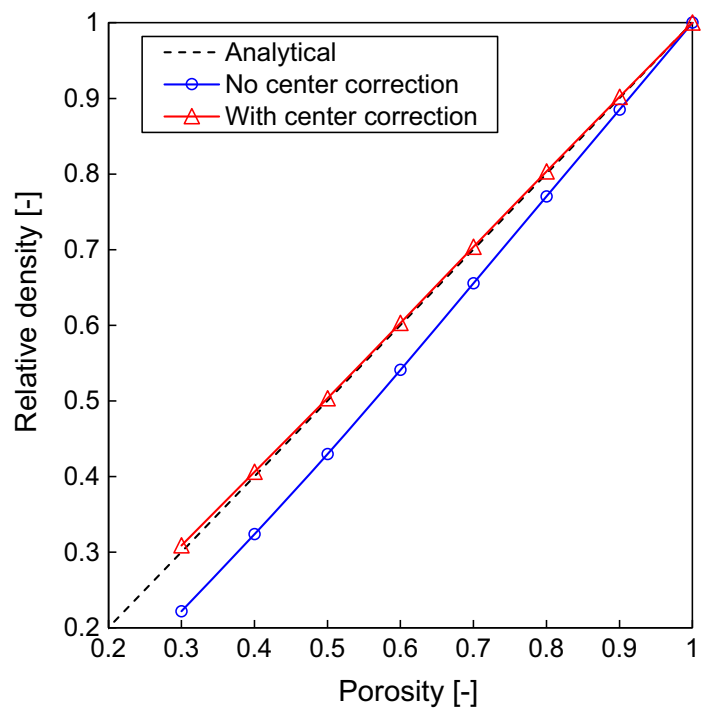

sity could be accurately mapped to the reference state with the help of the central weight correction. Consequently, the relative density is written as

$$
\rho_{\mathrm{rel}}=\frac{n_{\mathrm{rel}}}{\varepsilon}=\frac{1}{\varepsilon} \frac{n_{\varepsilon}+\alpha_{\varepsilon}}{n_{0}+\alpha_{0}}
$$

which is used to compute the pressure of weakly compressible fluids by the equation of state (7). It is worth noting that although derivations are for Eq. (5), the same procedure is also applicable to other MPS weight functions that are integrable in dimensions higher than two. 


\subsection{Balanced Gradient Model}

The pressure gradient term plays an important role in modeling the fluid interaction with porous structures. However, the symmetric gradient model (10) is not suitable for such flow cases, which disturbs the hydrostatic balance across a vertical porous boundary. The first row of Fig. 11 gives an example of how the inappropriate model may spoil the numerical solution. In this test, the computational domain is divided into the left part $(\varepsilon=1)$ and the right part ( $\varepsilon=0.49$ ). The fluid surface is uneven on different sides. In fact, it is observed that fluid particles are moving from the bottom of the free region into the porous region and flowing back over the cascade on the top. The spurious circulation across the porous boundary seems to be quasi-steady and ceaseless.

The reason of the unphysical result might be qualitatively explained by the unequal distribution of particles in adjacent domains. To facilitate the discussion, we substitute a constant field $p=\phi$ into Eq. (10), which becomes

$$
\langle\nabla \phi\rangle_{i}=2 \phi \cdot \frac{D}{n_{0, g}} \sum_{j \neq i}\left[\frac{\mathbf{x}_{i j}}{r_{i j}^{2}} w_{g}\left(r_{i j}\right)\right] .
$$

Let us consider a particle near the boundary. The equation is split by the contributions from different domains:

$$
\langle\nabla \phi\rangle_{i}=2 \phi \frac{D}{n_{0, g}}\left[\sum_{\text {free }} \frac{\mathbf{x}_{i j}}{r_{i j}^{2}} w_{g}\left(r_{i j}\right)+\sum_{\text {porous }} \frac{\mathbf{x}_{i j}}{r_{i j}^{2}} w_{g}\left(r_{i j}\right)\right] .
$$

Apparently, the first term and the second term in the bracket cannot be canceled by each other, as there will be more particles in the free side than in the porous side (Sect. 3.3). The residual is likely to be pointing to the free region where the particle distribution is denser, which partly explains how the spurious behavior is generated.

To solve this problem, a suitable gradient model must be used. As a point of departure, our development is based on the standard gradient model proposed by (Koshizuka et al. 1998):

$$
\langle\nabla p\rangle_{i}=\frac{D}{n_{0, g}} \sum_{j \neq i}\left[\frac{p_{j}-p_{i, \min }}{r_{i j}^{2}}\left(\mathbf{x}_{j}-\mathbf{x}_{i}\right) w_{g}\left(r_{i j}\right)\right] .
$$

Herein, $p_{i \text { min }}$ is the minimal pressure in the vicinity of the $i$ th particle including itself, i.e.,

$$
p_{i, \min }=\min _{j}\left\{p_{j} \mid r_{i j}<r_{e}\right\}
$$

which stabilizes the numerical model by eliminating tensile actions between particles. Being Taylor series consistent (Duan et al. 2017), the standard gradient model does not suffer from the previous problem (at least for a constant field). In this study, a corrected form of the standard model is adopted:

$$
\langle\nabla p\rangle_{i}=\frac{D}{n_{0, g}} \sum_{j \neq i}\left[\frac{p_{j}-p_{i, \min }}{r_{i j}^{2}}\left(\mathbf{x}_{j}-\mathbf{x}_{i}\right) \frac{w_{g}\left(r_{i j}\right)}{\varepsilon_{i j}}\right] .
$$

Herein, $\varepsilon_{i j}$ is a correction factor applied to the weight function to treat the influence of porosity. A simple and effective choice is to set

$$
\varepsilon_{i j}=\varepsilon_{j}
$$


Equation (28) with factor (29) is referred to as the corrected gradient model in the remaining part of this paper. In addition, there also exist other intuitive definitions of $\varepsilon_{i j}$, for example:

(a) $\varepsilon_{i j}=1$, which is equal to the standard model;

(b) $\quad \varepsilon_{i j}=\varepsilon_{i}$, i.e., to use the porosity of the center particle;

(c) $\varepsilon_{i j}=2 \varepsilon_{i} \varepsilon_{j} /\left(\varepsilon_{i}+\varepsilon_{j}\right)$, i.e., to use harmonic average of the porosities.

The effects of those variants will be examined later in this paper.

\subsection{Boundary Conditions}

For simplicity, only straight walls are considered in this study. The mirror particle technique (Morris et al. 1997) is used for wall boundary conditions, in which image particles are generated at mirror positions dynamically. Their velocities and positions are frozen in each computational step to model fixed walls.

The free surface boundary condition is treated by using the same approach as previous studies (Yamada and Sakai 2013; Sun et al. 2014). Surface particles are detected by deficiency in particle number density. In this study, an MPS particle is considered to be on the free surface if its relative density [Eq. (23)] is lower than one. Then, its pressure will be set to zero.

\subsection{Solution Algorithm}

The overall time-stepping algorithm is briefed as follows. At the $n$th computational step, the state variables of MPS particles, including their positions $\boldsymbol{x}^{n}$ and velocities $\boldsymbol{u}^{n}$, are given, and the time step is $\Delta t$.

(a) Determine the porosity $\varepsilon$ for each particle (Sect. 3.2).

(b) Evaluate the drag force based on the current porosity and fluid velocity [Eqs. (3), (4)].

(c) Calculate the particle number density $n$ for each particle using Eq. (6).

(d) Compute the corrected relative density using the method [Eq. (23)] explained in Sect. 3.3 and derive the pressure field $p$ from the equation of state (7).

(e) Compute the pressure gradient term by using the corrected gradient model (28) in Sect. 3.4.

(f) Compute other terms including the viscous force (8) and gravitational force, by which the forces exerted on fluid particles are obtained.

(g) Update the particle velocity and position by using the symplectic Euler scheme:

$$
\begin{aligned}
& \boldsymbol{u}^{n+1}=\boldsymbol{u}^{n}+\Delta t \dot{\boldsymbol{u}} \\
& \mathbf{x}^{n+1}=\mathbf{x}^{n}+\Delta t \boldsymbol{u}^{n+1} .
\end{aligned}
$$

It is noted that particle streaming is based on the actual velocity, rather than the average Darcy velocity.

The computational time step is limited by the transportation time (i.e., the CFL condition) of the weakly compressible MPS model (Sun et al. 2014):

$$
\Delta t<\frac{r_{e}}{|\boldsymbol{u}|_{\max }+c} \text {. }
$$


Again, it is noted that the artificial sound speed $c$ should be chosen properly to avoid restricting the time step. The second limitation is due to the fluid viscosity (Sun et al. 2012):

$$
\Delta t<\frac{\Delta x^{2}}{\mu / \rho} .
$$

For flow systems considered in this study, the kinematic viscosity (i.e., the ratio $\mu / \rho$ ) is relatively low and does not affect the stability in a significant way. Semi-implicit fractional step scheme can be used to eliminate this restriction. The third stability condition that has been rarely mentioned by previous studies arises from the drag force which is treated as an explicit source term in the momentum equation. By solving an ordinary differential equation of particle velocity subject to the drag (3), we find the following criterion based on the relaxation time of velocity decay:

$$
\Delta t<\frac{\rho}{A+B|\boldsymbol{U}|_{\max }} .
$$

Regarding those limitations as heuristic guides, proper time steps are set to ensure the stability of numerical simulations. The present time-stepping algorithm can also be adapted to work with a higher-order temporal integration scheme or variable time steps.

\section{Results and Discussion}

In this section, validation tests are performed to examine the validity of the new MPS model. A volume-of-fluid (VOF) code published previously (Sun and Sakai 2015, 2016) is used for reference as mesh-based numerical solution. It is intended to verify the present model by showing agreement with a well-established Eulerian VOF data. The improvements in volume conservation and hydrostatic balance are highlighted through comparison with existing MPS models.

\subsection{Fluid Saturating Porous Zone}

The first problem simulates fluid penetrating a porous region under constant gravity, which is schematically shown in Fig. 6. The computational domain is $0.9 \mathrm{~m}$ in length and $0.3 \mathrm{~m}$ in width and depth, respectively. Initially, one-third of the domain is filled by water ( $\rho=$ $1000 \mathrm{~kg} / \mathrm{m}^{3}$ and $\mu=0.001 \mathrm{~Pa} \mathrm{~s}$ ) and the rest of the domain is occupied by porous media whose porosity is $\varepsilon=0.5$ and effective diameter is $d_{\mathrm{p}}=2 \mathrm{~mm}$. When the simulation begins, the water flows into the porous region under the rightward gravity $\left(g=9.8 \mathrm{~m} / \mathrm{s}^{2}\right)$. It is thus expected that the water will saturate the entire porous region finally, which is required by the law of volume conservation. For this quasi-1D problem, the particle size is $\Delta x=0.02 \mathrm{~m}$, or 3375 MPS particles in total. The artificial sound speed is set to $c=20 \mathrm{~m} / \mathrm{s}$ to keep the fluid nearly incompressible. The time step is fixed by $\Delta t=10^{-5} \mathrm{~s}$. Those physical properties and

Fig. 6 Schematic diagram of the initial setting up of the simulation for fluid entering a porous zone

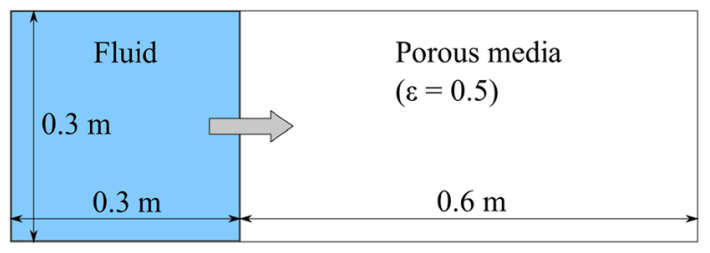


Table 1 Computational conditions of the simulation

\begin{tabular}{ll}
\hline Fluid density $\left(\mathrm{kg} / \mathrm{m}^{3}\right)$ & 1000 \\
Fluid viscosity $(\mathrm{Pa} \mathrm{s})$ & 0.001 \\
Porosity $(-)$ & 0.5 \\
Effective diameter $(\mathrm{mm})$ & 2.0 \\
Particle size $(\mathrm{m})$ & 0.02 \\
Particle number $(-)$ & 3375 \\
Artificial sound speed $(\mathrm{m} / \mathrm{s})$ & 20 \\
Time step (s) & $10^{-5}$ \\
\hline
\end{tabular}
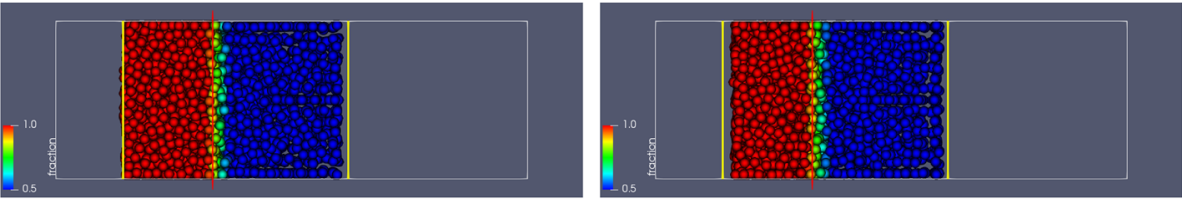

$\mathrm{t}=1.0 \mathrm{~s}$
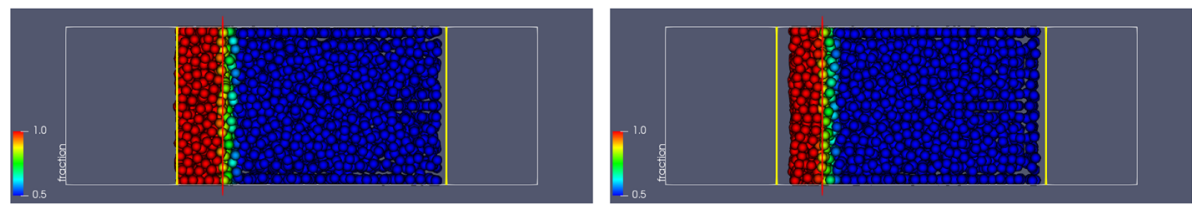

$\mathrm{t}=2.0 \mathrm{~s}$
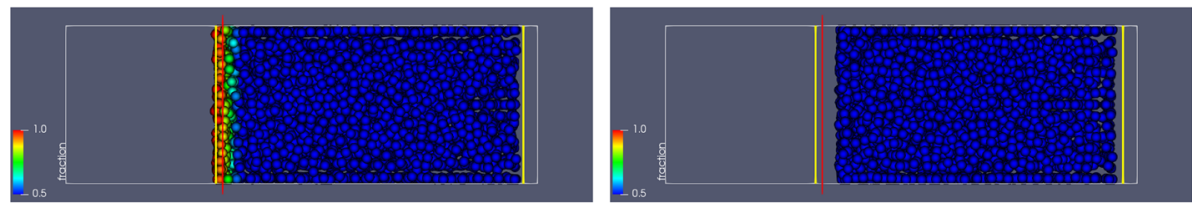

$\mathrm{t}=3.0 \mathrm{~s}$
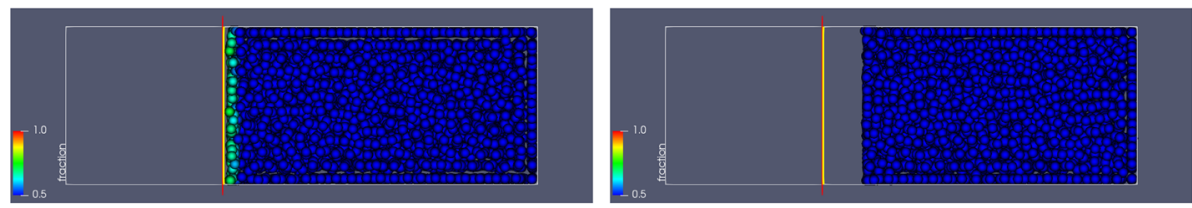

(a)

(b)

Fig. 7 Snapshots of simulation results at time instants $t=1,2,3,4 \mathrm{~s}$, respectively. The left column shows results with the central weight correction, and the right column shows those by existing model without correction. The red line is the boundary between open domain and porous domain. The light yellow lines show the VOF interfaces. a With central weight correction, $\mathbf{b}$ without correction

numerical parameters are listed in Table 1 . The VOF code for verification is executed with similar resolutions and conditions.

Figure 7 shows a series of snapshots of the motions when the fluid is entering the porous media, in which the MPS particles are colored by their porosity values and the interface positions obtained by the VOF method are plotted as yellow lines. From Fig. 7a, it can be confirmed that the proposed MPS model with central weight correction gives results in good 
Fig. 8 Temporal variations of the positions of the front surface and the back surface. Note that the length of error bars shows the size of MPS particle

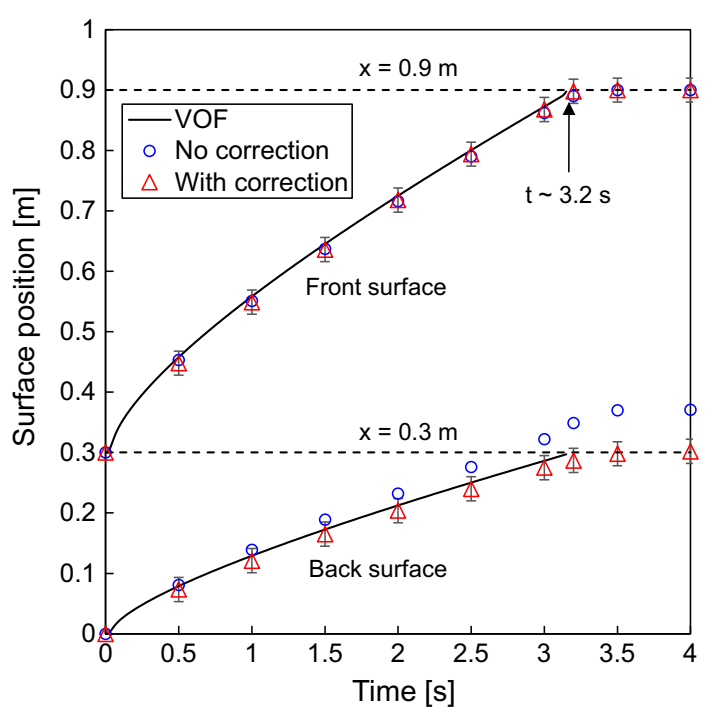

agreement with the VOF solution. The macroscopic fluid volume expands and fits into the porous zone properly at $t=4 \mathrm{~s}$. Without the correction, Fig. $7 \mathrm{~b}$ demonstrates that the final fluid volume is collapsed significantly, which fails to satisfy the conservation law. Figure 8 plots the positions of the front and rear surfaces against time. Note that the length of error bars indicates the MPS particle size which introduces uncertainty when measuring the surface position. By using the proposed model, the advancement of both surfaces well agrees with the reference data. The bottom position is reached around $t=3.2 \mathrm{~s}$. On the other hand, existing MPS model gives reasonable prediction of the front surface motion, but it fails to yield accurate results of the back surface even for such a relatively simple test. Hence, it is shown that the central weight correction developed in this study is a useful technique to enforce the volume conservation.

\subsection{Equilibrium Between Free and Porous Regions}

The second test problem focuses on fluid-porous structure interactions involving a vertical porous boundary. Consider a tank with a length of $0.59 \mathrm{~m}$ and width of $0.1 \mathrm{~m}$. A column of water is initially placed next to the left wall, whose dimensions are $0.28 \mathrm{~m}$ by $0.25 \mathrm{~m}$ by $0.1 \mathrm{~m}$. The deck of the tank is dry. The right-half side of the tank is filled with porous solid (porosity $\varepsilon=0.49$ and effective diameter $d_{\mathrm{p}}=15.9 \mathrm{~mm}$ ). The gravity $\left(g=9.8 \mathrm{~m} / \mathrm{s}^{2}\right)$ points downward. Once released, the water column flows along the deck into the porous bed. This problem is chosen because it requires the pressure gradient to be well balanced between the free and porous regions. The particle size is $\Delta x=0.01 \mathrm{~m}$ and the total particle number is 7000. Other numerical parameters are same as those used in the previous test, see Table 2 for summary. VOF-based simulation is carried out to obtain verification data, for which the grid spacing is set equal to the particle size.

Figure 9 shows representative snapshots of the simulation, in which the color contour is based on the porosity and the bold black line is the interface by the VOF method. It is observed that the water flows into the porous zone and forms a nearly flat surface by $t=$ $1.5 \mathrm{~s}$. Figure 10 shows the changes in surface positions at four gauges: the front of the water 
Table 2 Computational conditions of the simulation

\begin{tabular}{ll}
\hline Fluid density $\left(\mathrm{kg} / \mathrm{m}^{3}\right)$ & 1000 \\
Fluid viscosity $(\mathrm{Pa} \mathrm{s})$ & 0.001 \\
Porosity (-) & 0.49 \\
Effective diameter $(\mathrm{mm})$ & 15.9 \\
Particle size $(\mathrm{m})$ & 0.01 \\
Particle number $(-)$ & 7000 \\
Artificial sound speed $(\mathrm{m} / \mathrm{s})$ & 20 \\
Time step (s) & $10^{-5}$ \\
\hline
\end{tabular}
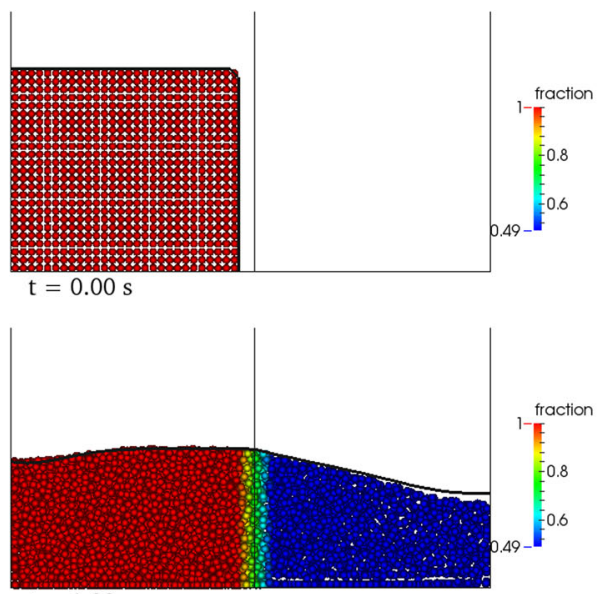

$\mathrm{t}=1.00 \mathrm{~s}$

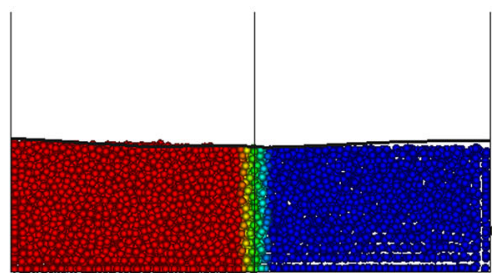

$\mathrm{t}=2.00 \mathrm{~s}$
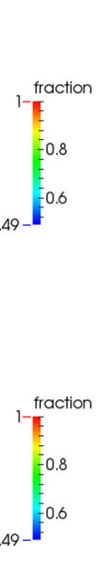

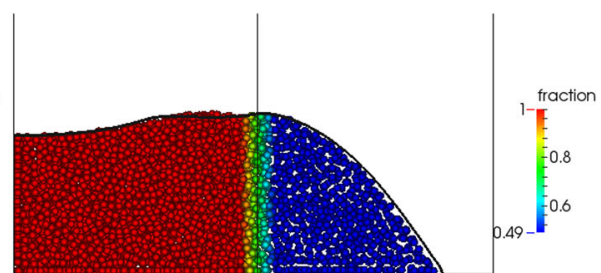

$\mathrm{t}=0.50 \mathrm{~s}$

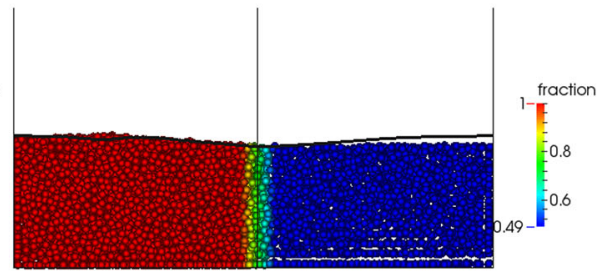

$\mathrm{t}=1.50 \mathrm{~s}$

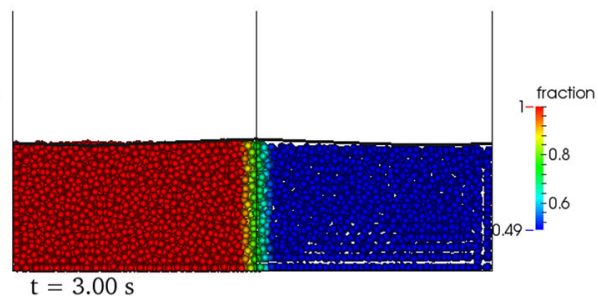

Fig. 9 Representative snapshots of simulation. MPS particles are colored by their porosity values. The black lines show the VOF interface

column, the heights at the left wall, the right wall, and the middle point. In general, the MPS results agree well with the VOF solutions within one particle size indicated by the error bars. Predictions of the front position (Fig. 10a) and the height at right wall (Fig. 10c) by the MPS model seem to be slightly lower than those by the VOF, which is possibly due to numerical smoothing near the porous boundary.

The influences of different MPS gradient models are investigated. Besides the corrected gradient model, the following models are used to solve the same test problem:

Case A: The symmetric gradient model, Eq. (10);

Case B: The standard gradient model, Eq. (26); 


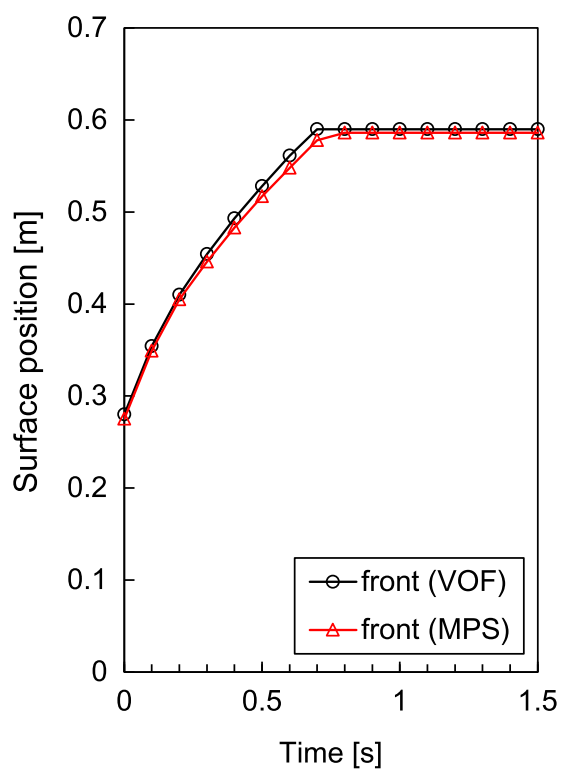

(a)

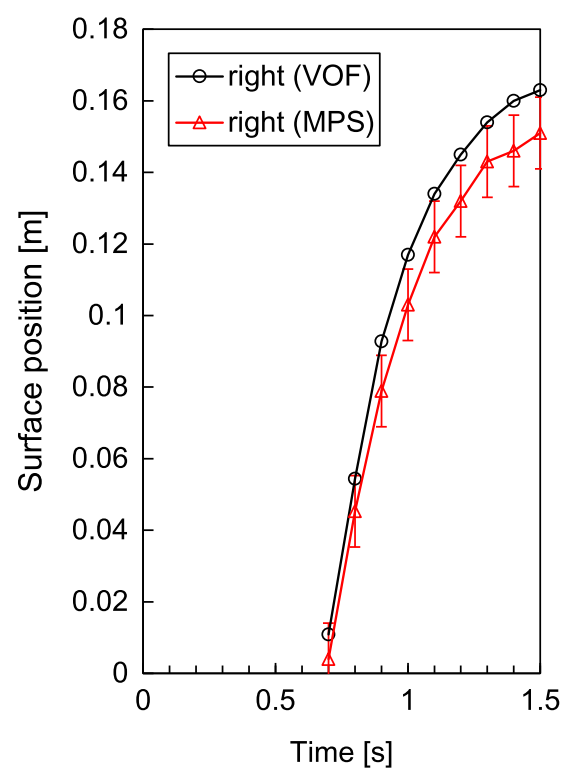

(c)

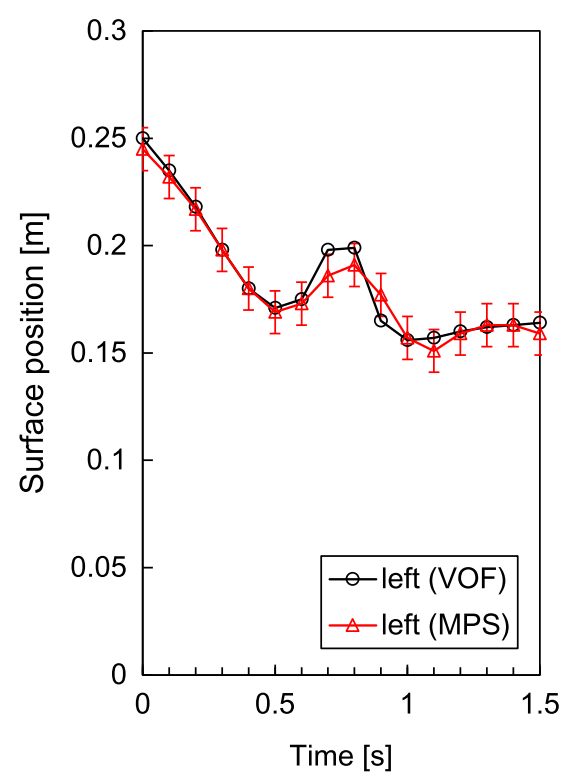

(b)

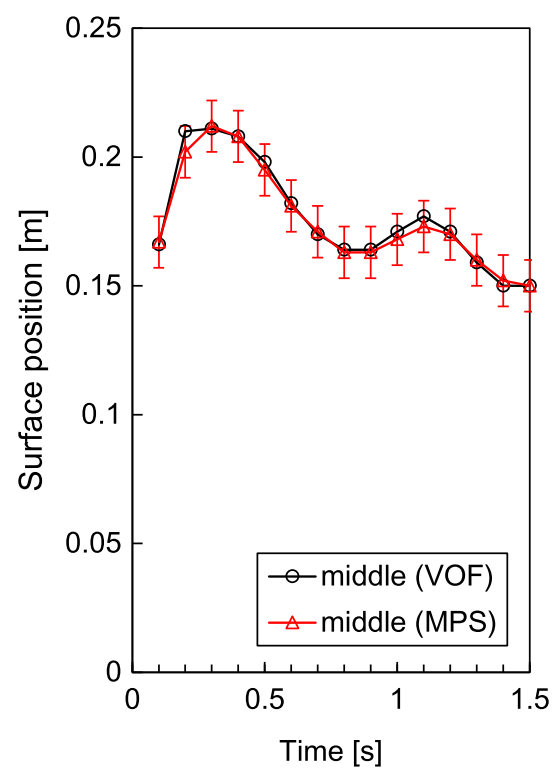

(d)

Fig. 10 Temporal variations of surface positions: a position of water front, $\mathbf{b}$ height at the left wall, $\mathbf{c}$ height at the right wall, and $\mathbf{d}$ height at the middle point. Note that the error bar indicates the MPS particle size 


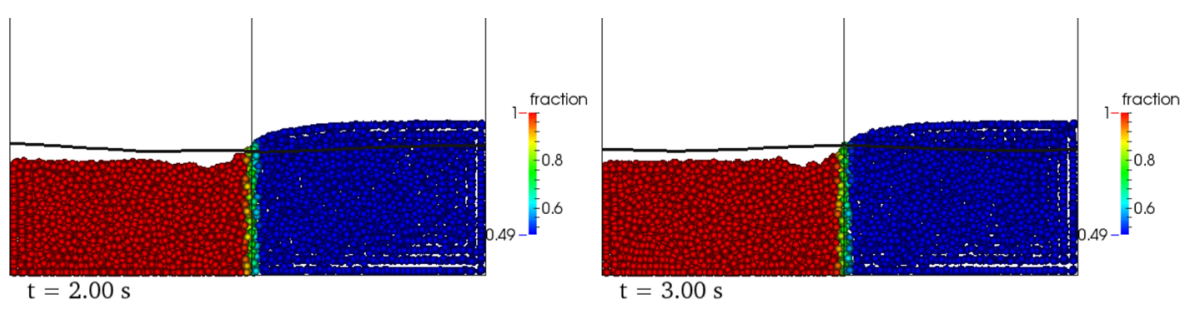

Case A: symmetric gradient model

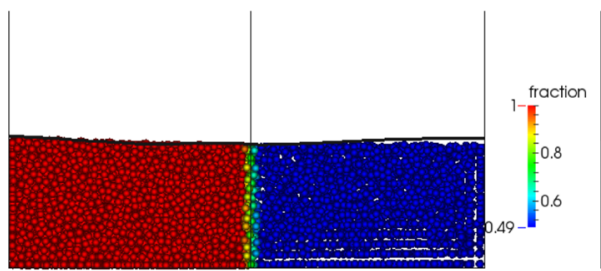

$\mathrm{t}=2.00 \mathrm{~s}$

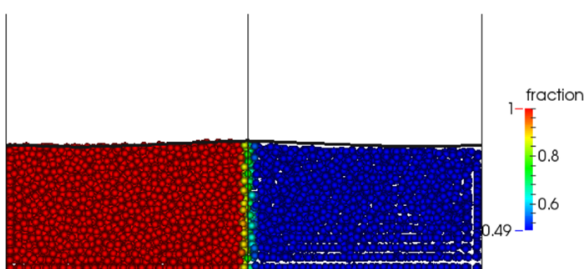

$\mathrm{t}=3.00 \mathrm{~s}$

Case B: standard gradient model

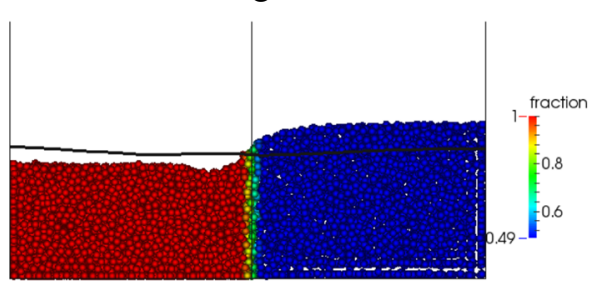

$\mathrm{t}=2.00 \mathrm{~s}$

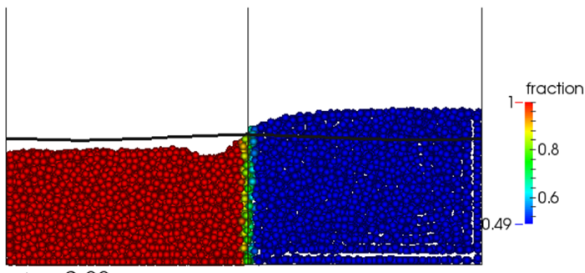

$\mathrm{t}=3.00 \mathrm{~s}$

Case C: corrected gradient model with central porosity $\varepsilon_{i}$

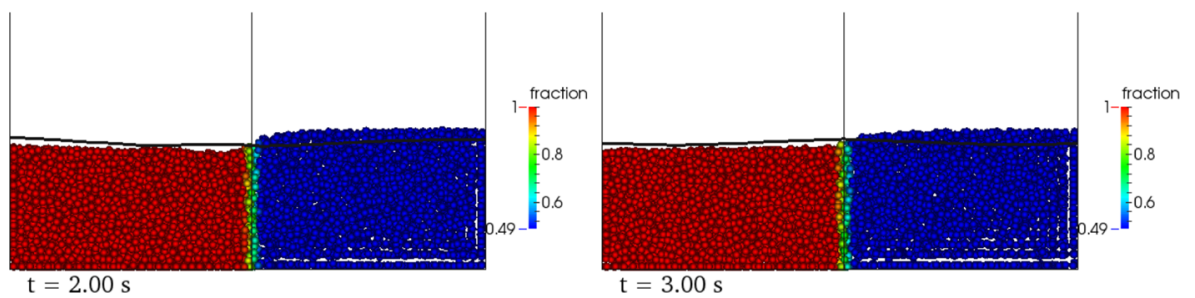

Case D: corrected gradient model with harmonic average of $\varepsilon_{i}$ and $\varepsilon_{j}$

Fig. 11 Comparison of numerical results obtained with different gradient models. For Cases A, C, D where inconsistent models are used, spurious behaviors of unequal surface heights are observed. The results of Case $\mathrm{B}$ using the standard gradient model seem to be reasonable. The black lines show VOF interfaces

Case C: Corrected gradient model using porosity of central particle, i.e., Eq. (28) with Eq. (30);

Case D: Corrected gradient model using harmonic mean of porosities, i.e., Eq. (28) with Eq. (31).

Snapshots of particle positions are shown in Fig. 11 at time $t=2.0 \mathrm{~s}$ and $3.0 \mathrm{~s}$. According to previous results, the water surface should be almost flat on both sides. However, unphysical 


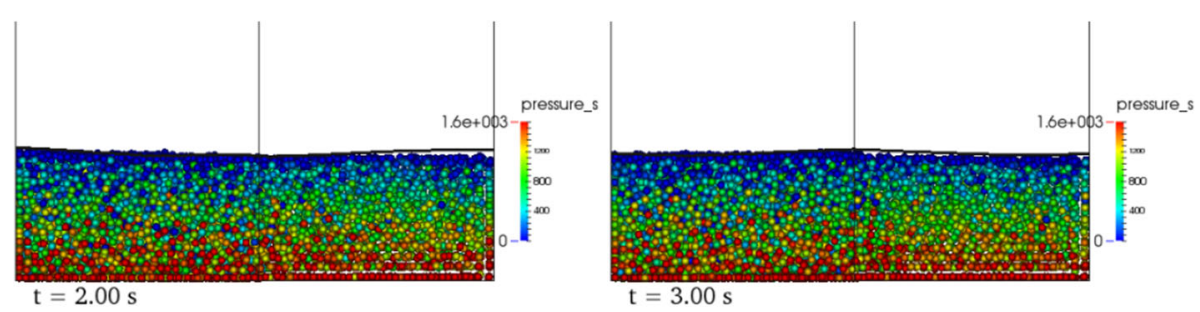

(a)

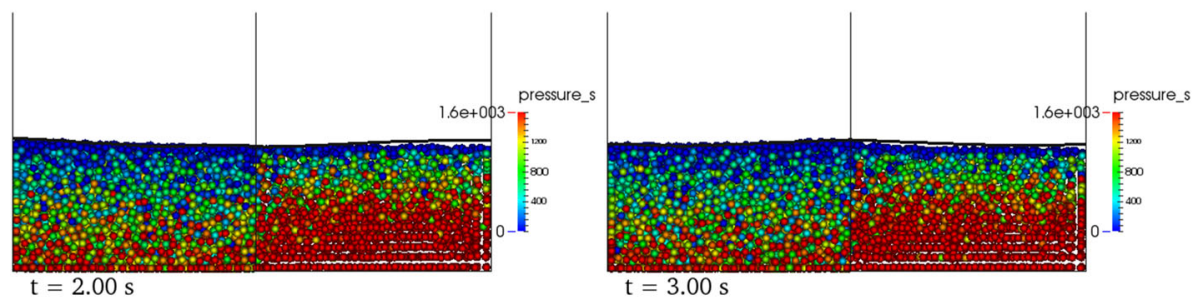

(b)

Fig. 12 Comparison of pressure solutions between $\mathbf{a}$ the corrected gradient model and $\mathbf{b}$ the standard gradient model. Although the particle configurations are similar, the pressure distributions differ. With the standard gradient model, the fluid pressure is higher in the porous region than in the free region. This unphysical effect is not found with the corrected gradient model

Fig. 13 Pressure distributions in fluid regions and porous regions. Data are obtained by averaging the particle pressure in vertical direction separately for different domains. The analytical distribution is based on an ideal hydrostatic state. The numerical results by the corrected gradient model agree well with the analytical equation, whereas those by the standard gradient model are overestimated in the porous region

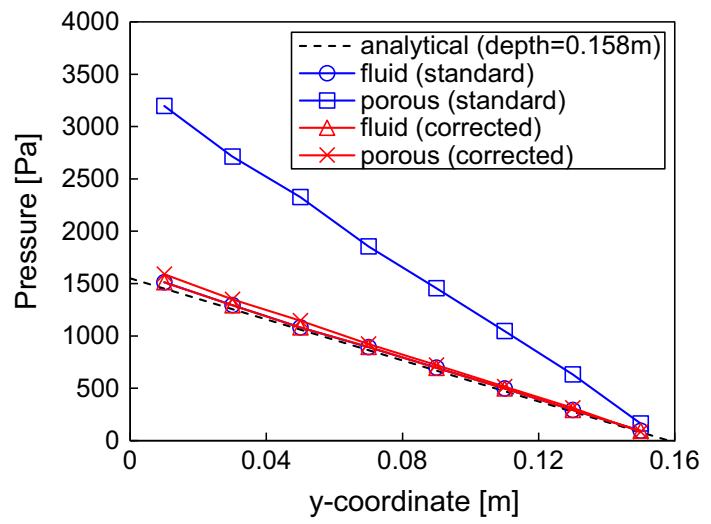

behaviors are observed in Cases A, C, and D, where the water surface in the porous side is significantly higher than the free side and the particles are circulating spuriously. The breakdown of balance between domains with different porosities must be ascribed to improper models of pressure gradient. Therefore, none of those models should be applied for simulating free surface flow-porous structure interactions.

On the other hand, the standard gradient model can also generate results that match the reference solutions satisfactorily in Case B. Despite their similarity in configuration of particles, the difference between the standard model and the corrected model resides in the pressure solution. Figure 12 shows the (almost hydrostatic) pressure fields obtained with those two models (note that noise is visible due to the explicit calculation of pressure). Physically, the pressure distribution is expected to be the same in both regions, as Fig. 12a 


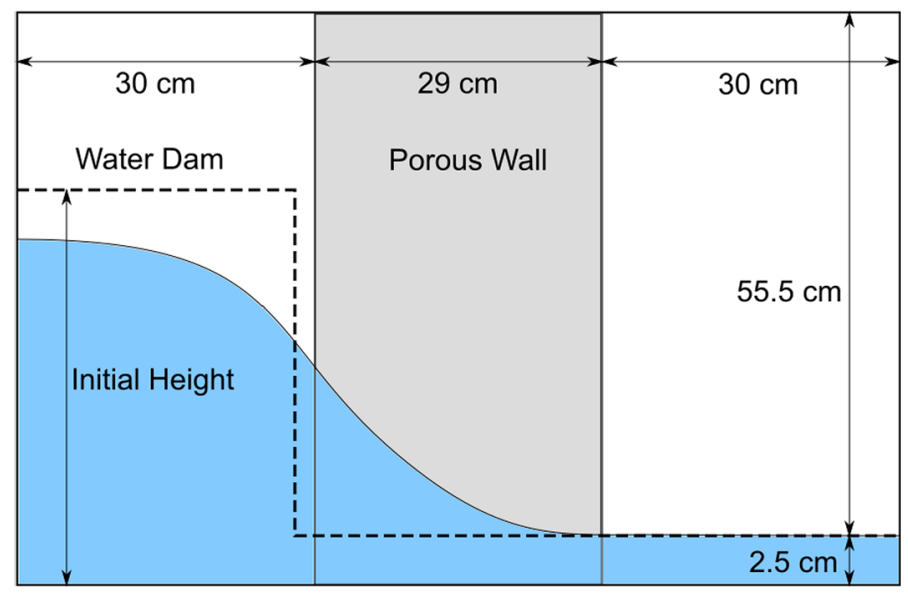

Fig. 14 Schematic diagram of the dam break flow through porous barrier

Table 3 Computational conditions of the dam break flow problem

\begin{tabular}{lll}
\hline & Case A (crashed rock) & Case B (glass beads) \\
\hline Fluid density $\left(\mathrm{kg} / \mathrm{m}^{3}\right)$ & 1000 & \\
Fluid viscosity $(\mathrm{Pa} \mathrm{s})$ & 0.001 & 0.39 \\
Porosity $(-)$ & 0.49 & 3.0 \\
Effective diameter $(\mathrm{mm})$ & 15.9 & 15 \\
Dam height $(\mathrm{cm})$ & 25 & 21131 \\
Particle size $(\mathrm{mm})$ & 5.0 & \\
Particle number $(-)$ & 32621 & \\
Artificial sound speed $(\mathrm{m} / \mathrm{s})$ & 20 & \\
Time step $(\mathrm{s})$ & $10^{-5}$ &
\end{tabular}

with the corrected gradient model. However, in Fig. 12b using the standard model, the fluid pressure is unnaturally higher in the porous region than in the free region. This problem is more clearly shown in Fig. 13, in which the vertical pressure distributions are plotted separately for the free part and the porous part. In the standard case, pressure within the porous region is overestimated by almost two times the free pressure as well as the analytical hydrostatic pressure, which is an evidence of lacking numerical consistency. The corrected gradient model does not suffer from this problem.

Therefore, the corrected gradient model [i.e., Eq. (28) combined with Eq. (29)] is shown to be the most suitable for simulating the interactions between flows and porous structures. It outperforms the previous symmetric gradient model with guarantee of balance between regions with different porosities. It is also more accurate in pressure distribution than the existing standard gradient model. 


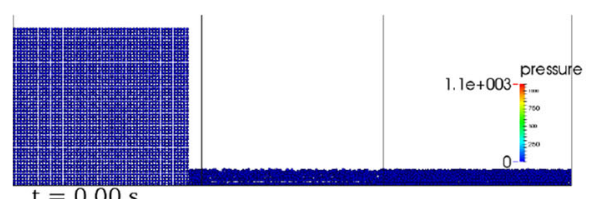

$\mathrm{t}=0.00 \mathrm{~s}$

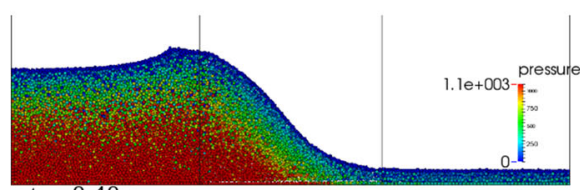

$\mathrm{t}=0.40 \mathrm{~s}$

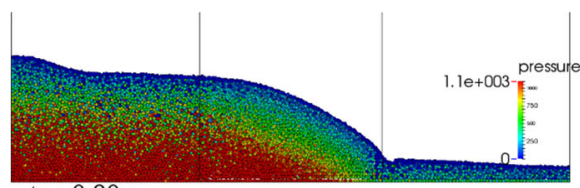

$\mathrm{t}=0.80 \mathrm{~s}$

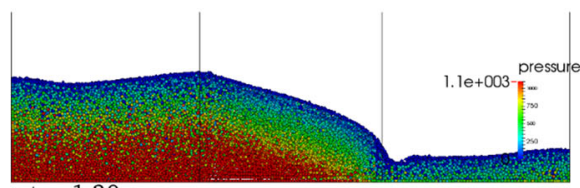

$\mathrm{t}=1.20 \mathrm{~s}$

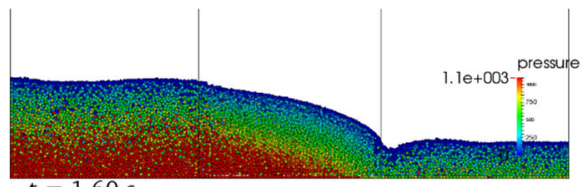

$\mathrm{t}=1.60 \mathrm{~s}$

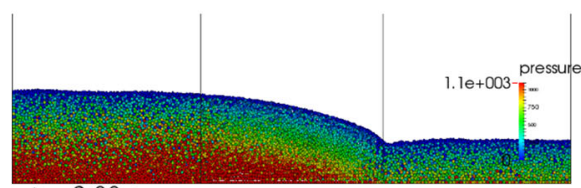

$\mathrm{t}=2.00 \mathrm{~s}$

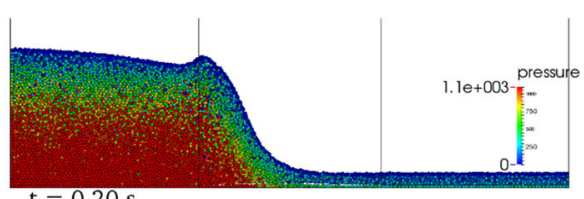

$\mathrm{t}=0.20 \mathrm{~s}$

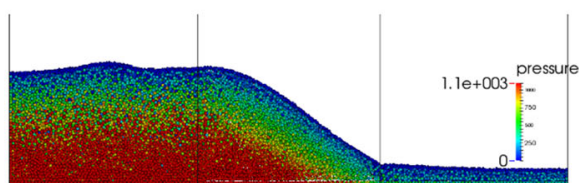

$\mathrm{t}=0.60 \mathrm{~s}$

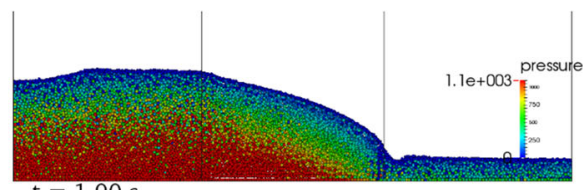

$\mathrm{t}=1.00 \mathrm{~s}$

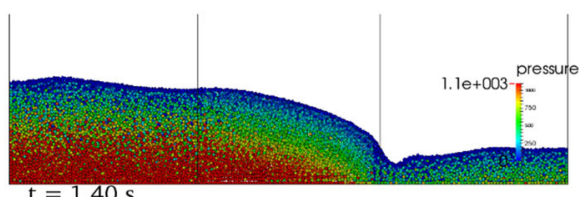

$\mathrm{t}=1.40 \mathrm{~s}$

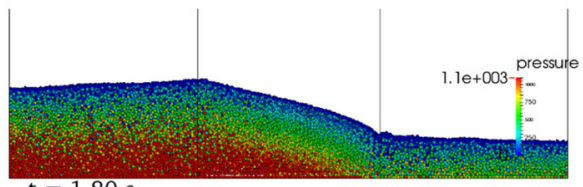

$\mathrm{t}=1.80 \mathrm{~s}$

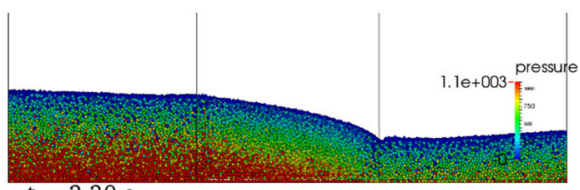

$\mathrm{t}=2.20 \mathrm{~s}$

Fig. 15 Snapshots of the dam break flow through a barrier of crashed rock. The scale of the pressure contour is based on a fully hydrostatic state

\subsection{Dam Break with Porous Barrier}

In the last example, the dam break problem with porous barriers described in Liu et al. (1999) is considered to validate the proposed MPS model. The schematic diagram of the problem layout is shown in Fig. 14. The tank is $89 \mathrm{~cm}$ long and $55.5 \mathrm{~cm}$ high, in the middle of which a porous block of $29 \mathrm{~cm}$ in length is placed. Initially, the ground water is $2.5 \mathrm{~cm}$ deep, and a water dam stands next to the left wall with a 2-cm gap from the porous barrier. Two test cases are simulated. In Case A, the porous block is composed by crashed rock with average porosity of 0.49 and effective granular diameter of $15.9 \mathrm{~mm}$, and the initial height of the water dam is $25 \mathrm{~cm}$. In Case B, fine glass beads are used resulting in average porosity of 0.39 and effective diameter equal to $3 \mathrm{~mm}$, and the dam height is reduced to $15 \mathrm{~cm}$. In both cases, the distance between MPS particles is set to $5 \mathrm{~mm}$, which is determined to readily resolve the ground water. The time step is set to $10^{-5} \mathrm{~s}$. The computational domain is extruded by $5 \mathrm{~cm}$ 


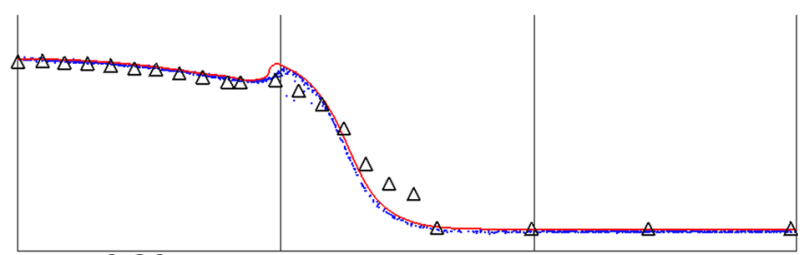

$\mathrm{t}=0.20 \mathrm{~s}$

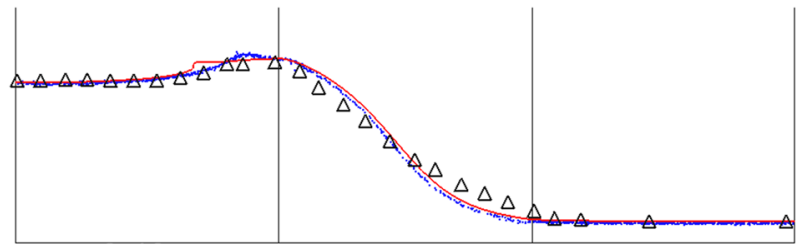

$\mathrm{t}=0.40 \mathrm{~s}$

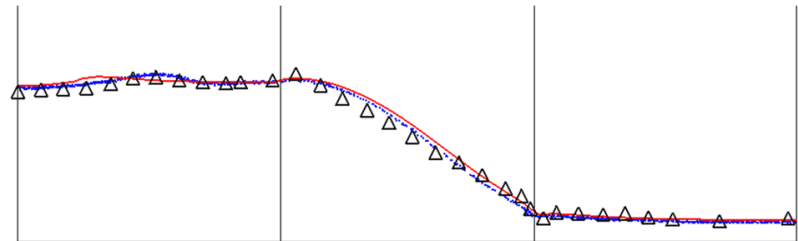

$\mathrm{t}=0.60 \mathrm{~s}$

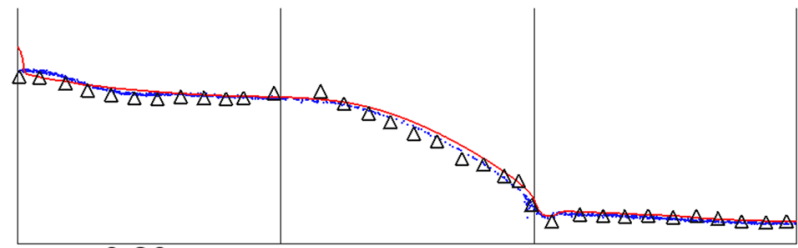

$\mathrm{t}=0.80 \mathrm{~s}$

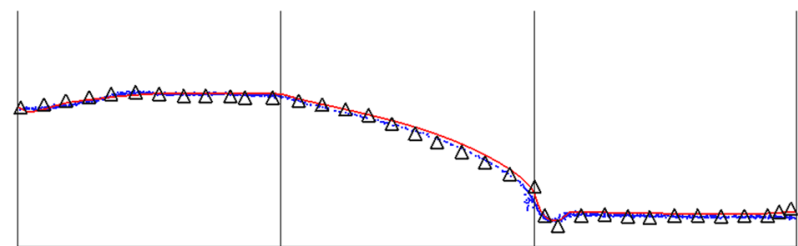

$\mathrm{t}=1.00 \mathrm{~s}$

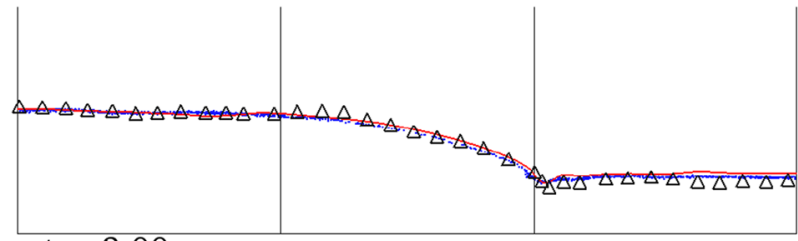

$\mathrm{t}=2.00 \mathrm{~s}$

Fig. 16 Comparison of free surface shapes for dam break flow through crashed rock barrier. Triangle markers are the experimental data by Liu et al. (1999). The red lines are from VOF simulation. The blue dots are present results, which are obtained by extracting the free surface particles with a threshold of 0.9 on the relative particle number density 


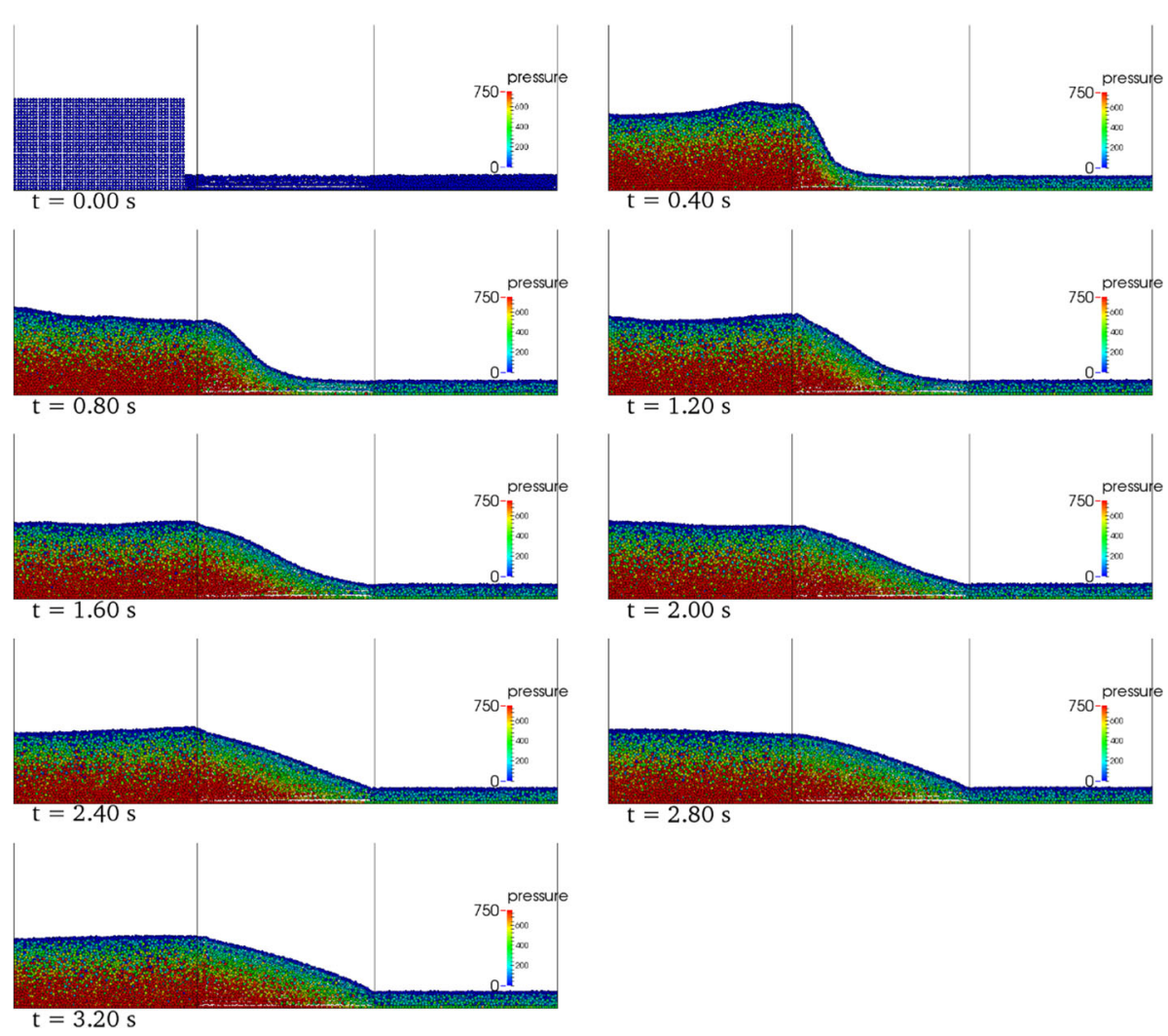

Fig. 17 Snapshots of the dam break flow through a barrier of glass beads. The scale of the pressure contour is based on a fully hydrostatic state

in the direction out of the paper to simulate this quasi-2D problem. Table 3 summarizes the computational conditions. Again, VOF simulations are conducted using similar parameters. The numerical results are compared with experimental data (Liu et al. 1999) for validation.

Figure 15 shows representative snapshots for Case A from initial state to $t=2.2 \mathrm{~s}$, in which MPS particles are colored by the pressure field. It is seen that dam break wave penetrates the porous barrier and forms an interface with rounded slope. A part of the wave hitting on the porous block is reflected $(t=0.4 \mathrm{~s})$ and reaches the left wall $(t=0.8 \mathrm{~s})$. Between $t=$ $1.0 \mathrm{~s}$ and $1.6 \mathrm{~s}$, a visible water jump can be found where the fluid leaves the porous region to the downstream flow. As time elapses, water flows through the porous barrier under pressure gradient, and the difference in surface heights between the left part and the right part becomes small gradually. Figure 16 compares the MPS results with the VOF interface solutions and the experimental data. MPS surface particles are extracted by using a threshold of 0.9 with respect to the relative particle number density, and they are rendered as blue dots. The red line shows the VOF interface, and the triangle markers are measured data in Liu et al. (1999). Their agreements are remarkable.

The flow behaviors in Case B are shown in Fig. 17. The flow motion is slower than that of the previous case because of lower dam height and stronger resistance due to low porosity and fine granularity. As a result, water jump is not observed in this case. Figure 18 


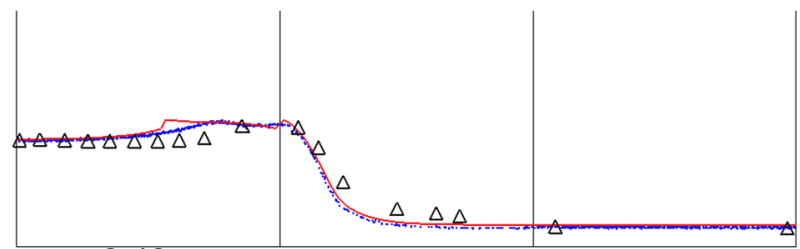

$\mathrm{t}=0.40 \mathrm{~s}$

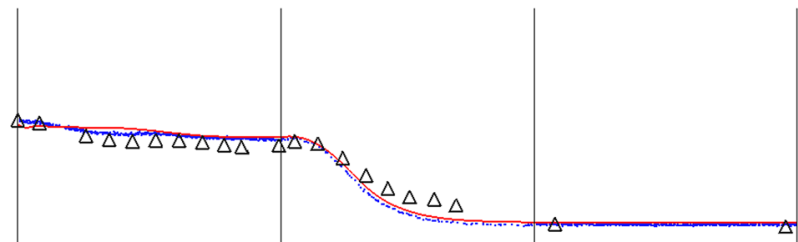

$\mathrm{t}=0.80 \mathrm{~s}$

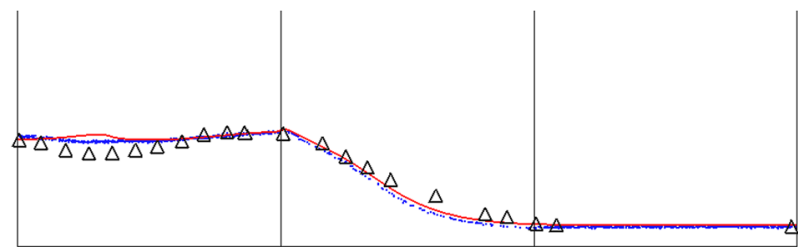

$\mathrm{t}=1.20 \mathrm{~s}$

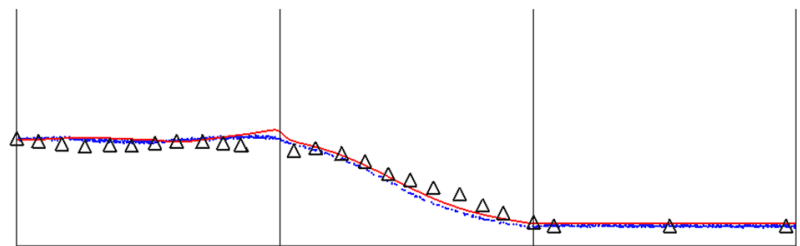

$\mathrm{t}=1.60 \mathrm{~s}$

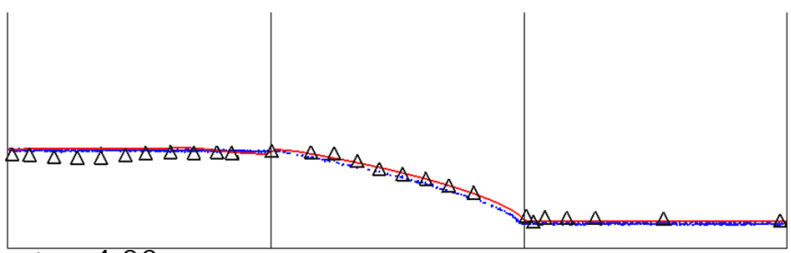

$\mathrm{t}=4.00 \mathrm{~s}$

Fig. 18 Comparison of free surface shapes for dam break flow through glass beads barrier. Triangle markers are the experimental data by Liu et al. (1999). The red lines are from VOF simulation. The blue dots are present results, which are obtained by extracting the free surface particles with a threshold of 0.9 on the relative particle number density

presents the comparison of MPS results, VOF results, and experimental data, from which good agreements can be confirmed. It is thus demonstrated that the proposed MPS model can simulate free surface flow-porous structure interactions accurately. 


\section{Conclusions}

In this study, an extension of the explicit MPS method was developed for the numerical simulation of free surface fluid flows interacting with porous structures. The present model is based on the local volume averaging technique. The drag resistance is given by a closure function of the local porosity and velocity. Two important techniques are proposed to improve the numerical results. The first one is the correction to the MPS particle number density by adding an equivalent central particle weight, which improves the volume conservation in porous regions where particles are dilute. The second point is to adopt a corrected pressure gradient model that guarantees the balance of hydrostatic pressure components between domains with different porosities. Numerical tests are performed to verify the new MPS model, whose results are shown to be in good agreements with the VOF method. It is confirmed that the proposed numerical techniques play key roles in conserving the macroscopic fluid volume and keeping well-balanced equilibrium for flows in porous media, which may be violated by simply adopting existing models. Finally, the new MPS model is applied to the dam break problem involving porous barrier, for which the simulation results agree remarkably with the experimental data. Hence, the validity and suitability of the present model are shown for Lagrangian simulations of macroscopic flow behaviors in porous media.

Acknowledgements This research was supported by the Initiatives for Atomic Energy Basic and Generic Strategic Research of the Ministry of Education, Culture, Science and Technology of Japan.

Open Access This article is distributed under the terms of the Creative Commons Attribution 4.0 International License (http://creativecommons.org/licenses/by/4.0/), which permits unrestricted use, distribution, and reproduction in any medium, provided you give appropriate credit to the original author(s) and the source, provide a link to the Creative Commons license, and indicate if changes were made.

\section{References}

Akbari, H.: Modified moving particle method for modeling wave interaction with multi layered porous structures. Coast. Eng. 89, 1-19 (2014). https://doi.org/10.1016/j.coastaleng.2014.03.004

Akbari, H., Namin, M.M.: Moving particle method for modeling wave interaction with porous structures. Coast. Eng. 74, 59-73 (2013). https://doi.org/10.1016/j.coastaleng.2012.12.002

Aly, A.M., Asai, M.: Three-dimensional incompressible smoothed particle hydrodynamics for simulating fluid flows through porous structures. Transp. Porous Media 110, 483-502 (2015). https://doi.org/10.1007/s1 1242-015-0568-8

Anderson, T.B., Jackson, R.: Fluid mechanical description of fluidized beds. Equations of motion. Ind. Eng. Chem. Fundam. 6, 527-539 (1967). https://doi.org/10.1021/i160024a007

Cundall, P.A., Strack, O.D.L.: A discrete numerical model for granular assemblies. Géotechnique 29, 47-65 (1979). https://doi.org/10.1680/geot.1979.29.1.47

Duan, G., Chen, B., Koshizuka, S., Xiang, H.: Stable multiphase moving particle semi-implicit method for incompressible interfacial flow. Comput. Methods Appl. Mech. Eng. 318, 636-666 (2017). https://doi.o $\mathrm{rg} / 10.1016 / \mathrm{j} . \mathrm{cma} .2017 .01 .002$

Ergun, S.: Fluid flow through packed columns. Chem. Eng. Prog. 48, 89-94 (1952)

Gómez-Gesteira, M., Cerqueiro, D., Crespo, C., Dalrymple, R.A.: Green water overtopping analyzed with a SPH model. Ocean Eng. 32, 223-238 (2005)

Hieu, P.D., Tanimoto, K.: Verification of a VOF-based two-phase flow model for wave breaking and wavestructure interactions. Ocean Eng. 33, 1565-1588 (2006). https://doi.org/10.1016/j.oceaneng.2005.10.0 13

Higuera, P., Lara, J.L., Losada, I.J.: Three-dimensional interaction of waves and porous coastal structures using OpenFOAM ${ }^{\circledR}$. Part I: formulation and validation. Coast. Eng. 83, 243-258 (2014). https://doi.or g/10.1016/j.coastaleng.2013.08.010

Hirt, C., Nichols, B.: Volume of fluid (VOF) method for the dynamics of free boundaries. J. Comput. Phys. 39, 201-225 (1981). https://doi.org/10.1016/0021-9991(81)90145-5 
Karim, M.F., Tanimoto, K., Hieu, P.D.: Modelling and simulation of wave transformation in porous structures using VOF based two-phase flow model. Appl. Math. Model. 33, 343-360 (2009). https://doi.org/10.10 16/j.apm.2007.11.016

Koshizuka, S., Oka, Y.: Moving-particle semi-implicit method for fragmentation of incompressible fluid. Nucl. Sci. Eng. 123, 421-434 (1996)

Koshizuka, S., Nobe, A., Oka, Y.: Numerical analysis of breaking waves using the moving particle semiimplicit method. Int. J. Numer. Methods Fluids 26, 751-769 (1998). https://doi.org/10.1002/(SICI)109 7-0363(19980415)26:7<751::AID-FLD671>3.0.CO;2-C

Liu, P.L.-F., Lin, P., Chang, K.-A., Sakakiyama, T.: Numerical modeling of wave interaction with porous structures. J. Waterw. Port Coast. Ocean Eng. 125, 322-330 (1999). https://doi.org/10.1061/(ASCE)073 3-950X(1999)125:6(322)

Monaghan, J.J.: Simulating free surface flows with SPH. J. Comput. Phys. 110, 399-406 (1994). https://doi. org/10.1006/jcph.1994.1034

Morris, J.P., Fox, P.J., Zhu, Y.: Modeling low Reynolds number incompressible flows using SPH. J. Comput. Phys. 136, 214-226 (1997). https://doi.org/10.1006/jcph.1997.5776

Nakayama, A., Kuwahara, F.: A macroscopic turbulence model for flow in a porous medium. J. Fluids Eng. 121, 427 (1999). https://doi.org/10.1115/1.2822227

Pahar, G., Dhar, A.: Modeling free-surface flow in porous media with modified incompressible SPH. Eng. Anal. Bound. Elem. 68, 75-85 (2016). https://doi.org/10.1016/j.enganabound.2016.04.001

Pahar, G., Dhar, A.: Numerical modelling of free-surface flow-porous media interaction using divergence-free moving particle semi-implicit method. Transp. Porous Media 118, 157-175 (2017). https://doi.org/10.1 007/s11242-017-0852-X

Rahimi-Gorji, M., Pourmehran, O., Gorji-Bandpy, M., Gorji, T.B.: CFD simulation of airflow behavior and particle transport and deposition in different breathing conditions through the realistic model of human airways. J. Mol. Liq. 209, 121-133 (2015). https://doi.org/10.1016/j.molliq.2015.05.031

Rahimi-Gorji, M., Gorji, T.B., Gorji-Bandpy, M.: Details of regional particle deposition and airflow structures in a realistic model of human tracheobronchial airways: two-phase flow simulation. Comput. Biol. Med. 74, 1-17 (2016). https://doi.org/10.1016/j.compbiomed.2016.04.017

Rahimi-Gorji, M., Ghajar, M., Kakaee, A.-H., Domiri Ganji, D.: Modeling of the air conditions effects on the power and fuel consumption of the SI engine using neural networks and regression. J. Braz. Soc. Mech. Sci. Eng. 39, 375-384 (2017). https://doi.org/10.1007/s40430-016-0539-1

Ren, B., Wen, H., Dong, P., Wang, Y.: Numerical simulation of wave interaction with porous structures using an improved smoothed particle hydrodynamic method. Coast. Eng. 88, 88-100 (2014). https://doi.org/1 0.1016/j.coastaleng.2014.02.006

Ren, B., Wen, H., Dong, P., Wang, Y.: Improved SPH simulation of wave motions and turbulent flows through porous media. Coast. Eng. 107, 14-27 (2016). https://doi.org/10.1016/j.coastaleng.2015.10.004

Sakai, M., Shigeto, Y., Sun, X., et al.: Lagrangian-Lagrangian modeling for a solid-liquid flow in a cylindrical tank. Chem. Eng. J. 200-202, 663-672 (2012). https://doi.org/10.1016/j.cej.2012.06.080

Shakibaeinia, A., Jin, Y.C.: A weakly compressible MPS method for modeling of open-boundary free-surface flow. Int. J. Numer. Methods Fluids 63, 1208-1232 (2010). https://doi.org/10.1002/fld.2132

Shao, S.: Incompressible SPH flow model for wave interactions with porous media. Coast. Eng. 57, 304-316 (2010). https://doi.org/10.1016/j.coastaleng.2009.10.012

Shibata, K., Koshizuka, S.: Numerical analysis of shipping water impact on a deck using a particle method. Ocean Eng. 34, 585-593 (2007). https://doi.org/10.1016/j.oceaneng.2005.12.012

Sun, X., Sakai, M.: Three-dimensional simulation of gas-solid-liquid flows using the DEM-VOF method. Chem. Eng. Sci. 134, 531-548 (2015). https://doi.org/10.1016/j.ces.2015.05.059

Sun, X., Sakai, M.: Numerical simulation of two-phase flows in complex geometries by using the volume-offluid/immersed-boundary method. Chem. Eng. Sci. 139, 221-240 (2016). https://doi.org/10.1016/j.ces. 2015.09.031

Sun, X., Sakai, M., Shibata, K., et al.: Numerical modeling on the discharged fluid flow from a glass melter by a Lagrangian approach. Nucl. Eng. Des. 248, 14-21 (2012)

Sun, X., Sakai, M., Yamada, Y.: Three-dimensional simulation of a solid-liquid flow by the DEM-SPH method. J. Comput. Phys. 248, 147-176 (2013). https://doi.org/10.1016/j.jcp.2013.04.019

Sun, X., Sakai, M., Sakai, M.-T., Yamada, Y.: A Lagrangian-Lagrangian coupled method for three-dimensional solid-liquid flows involving free surfaces in a rotating cylindrical tank. Chem. Eng. J. 246, 122-141 (2014). https://doi.org/10.1016/j.cej.2014.02.049

Takabatake, K., Sun, X., Sakai, M., et al.: Numerical study on a heat transfer model in a Lagrangian fluid dynamics simulation. Int. J. Heat Mass Transf. 103, 635-645 (2016). https://doi.org/10.1016/j.ijheatma sstransfer.2016.07.073 
Yamada, Y., Sakai, M.: Lagrangian-Lagrangian simulations of solid-liquid flows in a bead mill. Powder Technol. 239, 105-114 (2013). https://doi.org/10.1016/j.powtec.2013.01.030

Yamada, Y., Sakai, M., Mizutani, S., et al.: Numerical simulation of three-dimensional free-surface flows with explicit moving particle simulation method. Trans. At. Energy Soc. Jpn. 10, 185-193 (2011) 